\title{
SUBNORMAL SUBGROUPS IN DIRECT PRODUCTS OF GROUPS
}

\author{
PETER HAUCK \\ (Received 26 February 1985) \\ Communicated by H. Lausch
}

\begin{abstract}
A group $G$ is called normally (subnormally) detectable if the only normal (subnormal) subgroups in any direct product $G_{1} \times \cdots \times G_{n}$ of copies of $G$ are just the direct factors $G_{i}$. We give an internal characterization of finite subnormally detectable groups and obtain analogous results for associative rings and for Lie algebras. The main part of the paper deals with a study of normally detectable groups, where we verify a conjecture of $T$. O. Hawkes in a number of special cases.
\end{abstract}

1980 Mathematics subject classification (Amer. Math. Soc.): primary 20 E 15; secondary 20 D 35 , 16 A 99,17 B 05.

\section{Introduction}

During the Warwick Symposium on Soluble Groups in 1977, T. O. Hawkes asked the following question: under which conditions is a finite group "normally detectable"? Here a group $G$ is called normally detectable if in any direct product $G_{1} \times \cdots \times G_{n}$, where $G_{i} \cong G$ for $i=1, \ldots, n$, the direct factors $G_{1}, \ldots, G_{n}$ are the only normal subgroups isomorphic to $G$. Hawkes conjectured that this is the case if and only if $G$ is directly indecomposable and $\left|G / G^{\prime}\right|$ and $|Z(G)|$ are coprime. It is easy to see that these two properties are necessary for $G$ to be normally detectable. Even more, a well-known result of Remak [6] states that exactly under these conditions on $G$ the groups $G_{i}$ are the only direct factors of

(C) 1987 Australian Mathematical Society $0263-6115 / 87 \$ A 2.00+0.00$ 
$G_{1} \times \cdots \times G_{n}$ which are isomophic to $G$. Thus the validity of Hawkes' conjecture would mean a significant generalization of Remak's theorem. However, the motivation for studying this problem stems from the theory of finite soluble groups; Hawkes was led to the concept of a normally detectable group in connection with certain Fitting class constructions. For details the reader is referred to the forthcoming book of Doerk and Hawkes [3].

In this paper we shall deduce a series of partial results on Hawkes' conjecture. A final answer, however, is not obtained. We shall show that a finite group $G$ is normally detectable if either one of the conditions stated above is somewhat strengthened. For instance, if the coprimeness condition on $|Z(G)|$ and $\left|G / G^{\prime}\right|$ is required not only for the directly indecomposable group $G$ but also for certain non-nilpotent factor groups of $G$, then $G$ is normally detectable (Theorem 6.5).

On the other hand, if, apart from direct decompositions, some specific factorizations of the form $G=N S$, where $N$ is a non-nilpotent normal subgroup of $G$ and $S$ is a non-nilpotent subnormal complement for $N$ in $G$, are also excluded, then a group $G$ with $\left(|Z(G)|,\left|G / G^{\prime}\right|\right)=1$ is normally detectable (Theorem 6.2). We also show that Hawkes' conjecture is valid for groups which satisfy certain conditions on the structure of the automorphism group or on the size of the socle, etc. (Theorem 6.9). In the course of proving these results, we not only obtain information about the structure but also about the kind of embedding of a possible counterexample to Hawkes' conjecture (Theorem 6.8).

In contrast to the case of normally detectable groups, subnormally detectable groups (which are defined in the obvious way) are much easier to handle. It turns out that a finite group $G$ is subnormally detectable if and only if $G$ is directly indecomposable and $\left(\left|G / G^{\prime}\right|,|\operatorname{Fit}(G)|\right)=1$ (Theorem 4.2).

It is the transitivity of the subnormality relation which makes our method (iterated embeddings) work smoothly. Therefore, it is not surprising that similar arguments provide a short proof of the fact that a finite group $G \neq 1$ can never be characteristic in $G_{1} \times \cdots \times G_{n}\left(G_{i} \cong G\right.$ for $\left.i=1, \ldots, n\right)$ in case $n \geqslant 2$ (Proposition 7.1).

Finally, the result on subnormally detectable groups carries over to Lie algebras and associative rings; this is shown in Theorems 7.3 and 7.5.

The groups considered in this paper are not assumed to be finite; however, we have to impose certain finiteness conditions. Usually, the minimal condition on subnormal subgroups is needed. For most of the results on normally detectable groups we even have to assume that the group in question possesses a finite composition series.

Part of this work was done while the author enjoyed the hospitality of the University of Kentucky, U.S.A. and the Australian National University (as a Visiting Fellow). Special thanks are due to J. C. Beidleman, B. Brewster, and J. 
Cossey for several stimulating discussions on the subject of this paper, and to L. G. Kovács for some valuable suggestions.

\section{Notation}

Iterated commutators are assumed to be normed from the left. The notation $S \unlhd \unlhd G$ means that $S$ is a subnormal subgroup of $G$, and the defect of a subnormal subgroup $S$ of $G$ is defined to be the smallest integer $d$ such that there exists a chain $S=S_{0} \triangleleft S_{1} \triangleleft \cdots \triangleleft S_{d}=G$. For a subgroup $S$ of $G, S^{G}$ denotes the smallest normal subgroup of $G$ containing $S$ and $\operatorname{Core}_{G}(S)$ denotes the largest normal subgroup of $G$ contained in $S$. Fit $(G)$ stands for the Fitting subgroup of a group $G$ and $\operatorname{Soc}(G)$ for the socle of $G$, the subgroup generated by all minimal normal subgroups of $G$.

For a direct product $G_{1} \times \cdots \times G_{n}$, the natural projection onto $G_{i}$ is always denoted by $\pi_{i}$. Quite frequently we shall use $k$-tuples $J_{k}=\left(j_{1}, \ldots, j_{k}\right)$ of positive integers as indices for the factors in a direct product. Correspondingly, the projections are denoted by $\pi_{J_{k}}$. If $m$ and $k$ are positive integers, $\mathbf{m}^{k}$ stands for the cartesian product $\{1, \ldots, m\} \times \cdots \times\{1, \ldots, m\}$ ( $k$ times). For $J=\left(j_{1}, \ldots, j_{k}\right)$ $\in \mathbf{m}^{k}$ and $I=\left(i_{1}, \ldots, i_{t}\right) \in \mathbf{m}^{t}$, we write $(J, I)$ for the $(k+t)$-tuple $\left(j_{1}, \ldots, j_{k}\right.$, $\left.i_{1}, \ldots, i_{t}\right) \in \mathbf{m}^{k+t}$. When $k$-tuples $J=\left(j_{1}, \ldots, j_{k}\right)$ are used as indices for groups (or maps), $G_{J}, G_{j_{1}, \ldots, j_{k}}$, and $G_{J_{0}, j_{k}}$ denote the same object (where $J_{0}=$ $\left.\left(j_{1}, \ldots, j_{k-1}\right)\right)$. If $\sigma$ is a permutation on $\{1, \ldots, k\}$, and if $J=\left(j_{1}, \ldots, j_{k}\right) \in \mathbf{m}^{k}$, then $J \sigma$ is the $k$-tuple $\left(j_{1 \sigma}, \ldots, j_{k \sigma}\right)$.

Finally, $(a, b)$ is used as the notation for the greatest common divisor of the positive integers $a$ and $b$.

Group theoretical notations not explained here are consistent with those used by Huppert [4].

\section{Preparatory lemmas}

Groups considered in this paper are usually assumed to satisfy certain finiteness conditions. The minimal condition on subnormal subgroups is needed frequently, often together with the maximal condition on subnormal subgroups. Clearly, these are just the groups possessing a finite composition series. The purpose of this section is to collect some basic results on nilpotent groups with finiteness conditions and to prove some elementary statements about certain subgroups in direct products. 
The following lemma is well known (see e.g. [8, Section 12]).

3.1. LEMMA. (a) If $G$ is a group satisfying the maximal or minimal condition on subnormal subgroups, then $\operatorname{Fit}(G)$ is nilpotent and every nilpotent subnormal subgroup of $G$ is contained in $\operatorname{Fit}(G)$.

(b) If $G$ is a group with finite composition series, then every nilpotent factor group of a subnormal subgroup of $G$ is finite. In particular, $\operatorname{Fit}(G)$ is finite.

3.2. LeMMA. Let $G$ be a finitely generated nilpotent group and $N$ a non-trivial central subgroup of $G$. Then there exists a non-trivial homomorphism from $G$ into $N$.

Proof. Let $T$ be the torsion subgroup of $G$. If $G \neq T$, then $G / T$ has an infinite cyclic factor group. If $G=T$, then $G$ is finite. In both cases the assertion is clear.

The following lemma contains some basic facts on direct products which will be used frequently in the sequel.

3.3. Lemma. Let $G$ be a subgroup of $H_{1} \times \cdots \times H_{n}$ and let $I$ be a subset and $i$ an element of $\{1, \ldots, n\}$.

(a) If $G$ is subnormal in $G \pi_{1} \times \cdots \times G \pi_{n}$ of defect $d$, then $G \pi_{i} /\left(G \cap H_{i}\right)$ is nilpotent of class at most $d$.

(b) If $G$ is normal in $H_{1} \times \cdots \times H_{n}$, then $G \pi_{i} /\left(G \cap H_{i}\right) \leqslant Z\left(H_{i} /\left(G \cap H_{i}\right)\right)$.

(c) If $G$ is characteristic in $H_{1} \times \cdots \times H_{n}$, then $G \pi_{i} /\left(G \cap H_{i}\right)$ is centralized by every automorphism of $H_{i}$. In parts (d)-(f) let $H_{j} \cong G$ for all $j=1, \ldots, n$.

(d) Let $G$ satisfy the maximal or minimal condition on subnormal subgroups. If $G$ is subnormal in $H_{1} \times \cdots \times H_{n}$, and if there exists no non-trivial homomorphism from $G$ into $\operatorname{Fit}(G)$, then $G\left(\sum_{j \in I} \pi_{j}\right)=1$ if and only if $G \cap \times_{j \in I} H_{j}=1$.

(e) Let $G$ satisfy the maximal condition on subnormal subgroups. If $G$ is subnormal in $H_{1} \times \cdots \times H_{n}$, and if there exists no non-trivial homomorphism from $G$ into $Z(G)$, then $G\left(\sum_{j \in I} \pi_{j}\right) \leqslant \operatorname{Fit}\left(\times_{j \in I} H_{j}\right)$ if and only if $G \cap \times_{j \in I} H_{j}=1$.

(f) If $G$ is normal in $H_{1} \times \cdots \times H_{n}$, and if there exists no nontrivial homomorphism from $G$ into $Z(G)$, then $G\left(\sum_{j \in I} \pi_{j}\right)=1$ if and only if $G \cap \times_{j \in I} H_{j}=1$.

Proof. Clearly, $G \cap H_{i} \unlhd G \pi_{i}$.

(a) Let $h_{i 0}, h_{i 1}, \ldots, h_{i d} \in G \pi_{i}$ be arbitrary. There exist $h_{j k} \in G \pi_{j}, j=1, \ldots, n$, $j \neq i, k=1, \ldots, d$, such that $g_{k}=h_{i k} \Pi_{j \neq i} h_{j k} \in G$ for $k=1, \ldots, d$. Since $G$ is subnormal in $G \pi_{1} \times \cdots \times G \pi_{n}$ of defect $d$, we conclude that $\left[h_{i 0}, h_{i 1}, \ldots, h_{i d}\right]=$ $\left[h_{i 0}, g_{1}, \ldots, g_{d}\right] \in G \cap H_{i}$. 
(b) and (c) Let $h_{i} \in G \pi_{i}$ and $\alpha \in \operatorname{Inn}\left(H_{i}\right)$ or $\alpha \in \operatorname{Aut}\left(H_{i}\right)$, respectively. Then $\alpha$ induces an automorphism $\bar{\alpha}$ on $H_{1} \times \cdots \times H_{n}$ in the obvious way (and $\bar{\alpha}$ is inner if $\alpha$ is inner). Choose $h_{j} \in H_{j}, j=1, \ldots, n, j \neq i$, such that $g=\prod_{j=1}^{n} h_{j}$ $\in G$. Then $\left[h_{i}, \alpha\right]=[g, \bar{\alpha}] \in G \cap H_{i}$.

(d) and (e) If $G \cap \times_{j \in I} H_{j}=1$, then $G \pi_{j} \leqslant \operatorname{Fit}\left(H_{j}\right)$ for all $j \in I$, by (a) and 3.1(a). This proves (d) and one part of (e). For the second part of (e), suppose that $G\left(\Sigma_{j \in I} \pi_{j}\right)$ is nilpotent but that $G \cap \times_{j \in I} H_{j} \neq 1, G \cap \times_{j \in I} H_{j} \unlhd G\left(\sum_{j \in I} \pi_{j}\right)$ implies that $G \cap Z\left(G\left(\Sigma_{j \in I} \pi_{j}\right)\right) \neq 1$. As an epimorphic image of $G$, the nilpotent group $G\left(\Sigma_{j \in I} \pi_{j}\right)$ is finitely generated. By 3.2 , there exists a non-trivial homomorphism from $G\left(\sum_{j \in I} \pi_{j}\right)$ into $G \cap Z\left(G\left(\sum_{j \in I} \pi_{j}\right)\right) \leqslant Z(G)$, which is a contradiction.

(f) This follows from (b).

3.4. Definition. Let $G$ be a subgroup of $H_{1} \times \cdots \times H_{n}$. Then $G$ is called trivially embedded in $H_{1} \times \cdots \times H_{n}$ if $G \cap \times_{j \neq i} H_{j}=1$ for some $i \in$ $\{1, \ldots, n\}$.

The following observation is immediate from 3.3(d) and (f).

3.5. Remark. Let $G$ be a subnormal subgroup of $G_{1} \times \cdots \times G_{n}$, where $G_{i} \cong G$ for $i=1, \ldots, n$. Assume further that one of the following conditions is satisfied:

(a) $G$ satisfies the maximal or minimal condition on subnormal subgroups, and there exists no non-trivial homomorphism from $G$ into Fit $(G)$;

(b) $G$ is normal in $G_{1} \times \cdots \times G_{n}$, and there exists no non-trivial homomorphism from $G$ into $Z(G)$. Then the following statements are equivalent in pairs.

(i) $G$ is trivially embedded.

(ii) There exists $i \in\{1, \ldots, n\}$ such that $G \cap G_{j}=1$ for all $j \neq i$.

(iii) There exists $i \in\{1, \ldots, n\}$ such that $G \unlhd \unlhd G_{i}$.

Trivial subnormal embeddings are easy to describe.

3.6. Lemma. Let $H_{1}, \ldots, H_{n}$ be groups which satisfy the maximal or minimal condition on subnormal subgroups.

(a) If $G$ is trivially subnormally embedded in $H_{1} \times \cdots \times H_{n}$, i.e. $G \cap \times_{j \neq i} H_{j}$ $=1$ for some $i \in\{1, \ldots, n\}$, then $G \pi_{i} \cong G$, and there exist homomorphisms $\alpha_{j}: G \pi_{i} \rightarrow \operatorname{Fit}\left(H_{j}\right)$ for all $j \neq i$ such that $G=\left\{g_{i} \cdot \prod_{j \neq i} g_{i} \alpha_{j} \mid g_{i} \in G \pi_{i}\right\}$.

(b) For some $i \in\{1, \ldots, n\}$, let $G_{i}$ be a subgroup of $H_{i}$. For $j \neq i$, let $\alpha_{j}: G_{i} \rightarrow \operatorname{Fit}\left(H_{j}\right)$ be homomorphisms. Then the subgroup $G=\left\{g_{i} \cdot \prod_{j \neq i} g_{i} \alpha_{j} \mid g_{i} \in G_{i}\right\}$ of $H_{1} \times \cdots \times H_{n}$ is isomorphic to $G_{i}$, and $G \cap \times_{j \neq i} H_{j}=1$. Moreover, if $G_{i}$ is subnormal in $H_{i}$, then $G$ is subnormal in $H_{1} \times \cdots \times H_{n}$. 
Proof. The straightforward proof is left to the reader.

We conclude this section with a description of those subgroups of a direct product of isomorphic groups which are isomorphic to one of the direct factors. This result is due to Remak [7, Satz 3].

3.7. LEMMA (Remak). Let $G$ be a group with $G \cong G_{i}$ via isomorphisms $\varphi_{i}: G \rightarrow G_{i}, i=1, \ldots, n$. If $\alpha_{i}: G \rightarrow G, i=1, \ldots, n$, are homomorphisms with $\bigcap_{i=1}^{n}$ ker $\alpha_{i}=1$, then the subgroup $G_{0}=\left\{\prod_{i=1}^{n} g \alpha_{i} \varphi_{i} \mid g \in G\right\}$ of $G_{1}$ $\times \cdots \times G_{n}$ is isomorphic to $G$. Conversely, every subgroup of $G_{1} \times \cdots \times G_{n}$ which is isomorphic to $G$ is obtained in this manner.

\section{Subnormally detectable groups}

4.1. Definition. A group $G$ is called subnormally detectable if the following holds: whenever $G_{0}$ is subnormal in $G_{1} \times \cdots \times G_{n}$ for some positive integer $n$, where $G_{j} \cong G$ for $j=0,1, \ldots, n$, then $G_{0}=G_{i}$ for some $i \in\{1, \ldots, n\}$.

The following theorem characterizes those subnormally detectable groups that satisfy the minimal condition on subnormal subgroups.

4.2. TheOREM. Let $G$ be a group satisfying the minimal condition on subnormal subgroups. Then the following statements are equivalent:

(i) $G$ is subnormally detectable;

(ii) $G$ is directly indecomposable, and there exists no non-trivial homomorphism from $G$ into $\operatorname{Fit}(G)$.

Proof. We show first that (i) implies (ii). If $G=A \times B, A \neq 1 \neq B$, then let $A_{i} \cong A, B_{i} \cong B$, and $G_{i}=A_{i} \times B_{i}$ for $i=1$, 2. Since $G \cong A_{1} \times B_{2} \unlhd G_{1} \times G_{2}, G$ is not subnormally detectable. If $\alpha: G \rightarrow \operatorname{Fit}(G)$ is a non-trivial homomorphism, let $G_{0}=\{(g, g \alpha) \mid g \in G\} \leqslant G \times G$. Then $G_{0} \cong G$ and $\operatorname{ker} \alpha \times 1 \leqslant G_{0} \leqslant$ $G \times \operatorname{Fit}(G)$. By $3.1(a),(G \times \operatorname{Fit}(G)) /(\operatorname{ker} \alpha \times 1)$ is nilpotent. Hence $G_{0}$ is subnormal in $G \times G$. Since $G \alpha \neq 1, G$ is not subnormally detectable.

Now assume (ii) and suppose that $G$ is not subnormally detectable. Then there exist groups $G_{0}, G_{1}, \ldots, G_{n}(n \geqslant 2)$ isomorphic to $G$ such that $G_{0} \unlhd \unlhd G_{1} \times \cdots \times G_{n}$ 
and such that $G_{0} \pi_{i} \neq 1$ for $i=1, \ldots, n$. Among all such subnormal embeddings choose one with $n$ maximal. This is possible: by $3.3(\mathrm{~d}), G_{0} \pi_{j} \neq 1$ and $G_{0} \cap G_{j} \neq 1$ are equivalent; because of the minimal condition on normal subgroups, each $G_{0} \cap G_{j}$ contains a minimal normal subgroup of $G_{0}$; finally, by the minimal condition on normal subgroups again, $\operatorname{Soc}\left(G_{0}\right)$ is a direct product of finitely many minimal normal subgroups. We now define groups $G_{i j} \cong G, i, j=1, \ldots, n$, such that each $G_{i}$ is subnormally embedded in $G_{i 1} \times \cdots \times G_{i n}$ in the same way as $G_{0}$ is embedded in $G_{1} \times \cdots \times G_{n}$. Clearly then, $G_{0}$ is subnormal in ( $G_{11}$ $\left.\times \cdots \times G_{1 n}\right) \times \cdots \times\left(G_{n 1} \times \cdots \times G_{n n}\right)$. By the maximal choice of $n$, at least $n^{2}-n$ of the projections $G_{0} \pi_{i j}$ are trivial. But then $1 \neq G_{0} \pi_{i} \leqslant G_{0} \pi_{i 1}$ $\times \cdots \times G_{0} \pi_{i n}$ implies that for each $i$ there exists exactly one index $j(i)$ such that $G_{0} \pi_{i, j(i)} \neq 1$. It follows that

$$
G_{0} \unlhd \unlhd\left(G_{1} \cap G_{1, j(1)}\right) \times \cdots \times\left(G_{n} \cap G_{n, j(n)}\right) .
$$

Note that $G_{i} \cap G_{i, j(i)} \neq 1$ for all $i(3.3(\mathrm{~d}))$ and that $G_{0} \pi_{i}$ satisfies the minimal condition on subnormal subgroups (since $G_{0} \pi_{i} \unlhd \unlhd G_{i}$ ). Consequently, there exists some $m \in\{1, \ldots, n\}$ such that $G_{0} \pi_{m}$ is not isomorphic to a proper subnormal subgroup of any $G_{0} \pi_{i}, i=1, \ldots, n$. We note further that $G_{0} \pi_{i} \cong G_{j} \pi_{j i}$ for all $i, j=1, \ldots, n$ : this is immediate from the fact that $G_{0}$ is embedded in $G_{1} \times \cdots \times G_{n}$ in the same way as $G_{j}$ is embedded in $G_{j 1} \times \cdots \times G_{j n}$. Employing (*), we now obtain $G_{0} \pi_{m} \unlhd \unlhd G_{m} \cap G_{m, j(m)} \unlhd G_{m} \pi_{m, j(m)} \cong G_{0} \pi_{j(m)}$. By the choice of $m, G_{0} \pi_{m}$ is not isomorphic to a proper subnormal subgroup of $G_{0} \pi_{j(m)}$. Hence, $G_{m} \cap G_{m, j(m)}=G_{m} \pi_{m, j(m)}$. But this implies that $G_{m}=\left(G_{m} \cap G_{m, j(m)}\right) \times\left(G_{m} \cap\right.$ $\left.\times_{j \neq j(m)} G_{m j}\right)$, contradicting the indecomposability of $G_{m}$.

If we use the fact that for finite nilpotent groups $G$ the prime divisors of $|G|$ coincide with those of $\left|G / G^{\prime}\right|$, then the following corollary is an immediate consequence of Theorem 4.2.

4.3. Corollary. Let $G$ be a finite group. Then the following statements are equivalent:

(i) $G$ is subnormally detectable;

(ii) $G$ is directly indecomposable and $\left(\left|G / G^{\prime}\right|,|\operatorname{Fit}(G)|\right)=1$.

Theorem 4.2 also yields the following somewhat more general statement.

4.4. COROLLARY. Let $G$ be a group satisfying the minimal condition on subnormal subgroups. Assume that $G$ is directly indecomposable and that there exists no 
non-trivial homomorphism from $G$ into $\operatorname{Fit}(G)$. If $G$ is subnormal in $H_{1} \times \cdots \times H_{n}$, where each $H_{j}$ is isomorphic to a subnormal subgroup of $G$, then there exists $i \in\{1, \ldots, n\}$ such that $G=H_{i}$.

\section{Subnormal embeddings}

For our approach to Hawkes' problem on normally detectable groups it is necessary to obtain some general information about subnormal embeddings of a group $G$ (with finite composition series) in a direct product of groups isomorphic to $G$. The aim of this section is to provide a proof of the following result.

5.1. THEOREM. Let $G$ be a group with finite composition series. Assume further that $G_{0}$ is subnormal in $G_{1} \times \cdots \times G_{n}$, where $G_{j} \cong G$ for $j=0,1, \ldots, n$. Then one of the following holds:

(a) $G$ is nilpotent;

(b) $G_{0} \cap G_{i} \neq 1$ and $G_{0} \cap \times_{j \neq i} G_{j}=1$ for some $i \in\{1, \ldots, n\}$;

(c) $G_{0}$ is non-trivially subnormally embedded (in the sense of 3.4); in this case, $G=N S$, where $N \unlhd G, S \unlhd \unlhd G, N \cap S=1, N \neq 1, S$ is not nilpotent, and $S^{G} / \operatorname{Core}_{G}(S)$ is nilpotent; in particular, $[N, S] \leqslant \operatorname{Fit}(G)$.

Since in nilpotent groups all subgroups are subnormal, the embeddings considered in Theorem 5.1 are classified for nilpotent groups by Lemma 3.7.

The trivial embeddings of 5.1(b) are described in Lemma 3.6. In view of Theorem 4.2 one might be led to conjecture that all subnormal embeddings for directly indecomposable groups arise like those in 3.6. However, in 5.12 we give an example of a finite group $G$ (due to John Cossey) which shows that this is not the case (even if $Z(G)=1$ ); hence case (c) of Theorem 5.1 actually occurs.

On the other hand, it is easy to construct examples of groups $G$ (with trivial center) that satisfy the structural properties given in 5.1(c) without admitting a non-trivial subnormal embedding in a direct product of groups isomorphic to $G$. We note further that Theorem 5.1 also yields a proof of Theorem 4.2 for groups with finite composition series. However, this approach is more elaborate than the direct argument in Section 4.

To prove Theorem 5.1 we observe that $G_{0} \cap G_{j}=1$ for all $j=1, \ldots, n$ implies that $G$ is nilpotent (3.3(a)). Therefore, we can assume that the non-nilpotent group $G_{0}$ is non-trivially subnormally embedded in $G_{1} \times \cdots \times G_{n}$ and have to show that $G$ is factorized in the form given in 5.1(c). This will be accomplished in Theorem 5.10, where also a concrete description of $N$ and $S$ can be found. Actually, it is this description which is most essential for the investigations on normally detectable groups in Section 6 . 
First of all, however, we have to introduce some notation. We assume in the following that all groups considered possess a finite composition series, although the full strength of this assumption is not always needed.

5.2. Notation. Let $G$ be a non-nilpotent group with finite compoșition series. Suppose that $G_{0} \unlhd \unlhd G_{1} \times \cdots \times G_{n}$, where $G_{j} \cong G$ for $j=0,1, \ldots, n$. These hypotheses are tacitly assumed throughout 5.2 to 5.9.

(a) With $G_{1}, \ldots, G_{n}$ given, we define groups $G_{J_{l}} \cong G, l \in \mathbb{N}, J_{l} \in \mathbf{n}^{\prime}$, in such a way that $G_{J_{l}}$ is subnormally embedded in $G_{J_{l}, 1} \times \cdots \times G_{J_{l}, n}$ in the same way as $G_{0}$ is embedded in $G_{1} \times \cdots \times G_{n}$. Hence, for all $l \in \mathbb{N}$, $G_{0}$ is subnormal in $\times_{J_{l} \in{ }_{\pi}} G_{J_{I}}$, where we use the lexicographical ordering in $\mathbf{n}^{\prime}$.

(b) For $l \in \mathbb{N}$, define $\mathscr{B}_{l} \subseteq \mathbf{n}^{l}$ by $\mathscr{B}_{l}=\left\{J_{l} \in \mathbf{n}^{l} \mid G_{0} \pi_{J_{l}} \nless\right.$ Fit $\left.\left(G_{J_{l}}\right)\right\}$ and set $b_{l}=$ $\left|\mathscr{B}_{l}\right| \cdot\left(\pi_{J_{l}}\right.$ denotes the projection onto $G_{J_{l}}$; cf. Section 2.) By 3.3(a), $B_{l} \subseteq\left\{J_{l} \in\right.$ $\left.\mathbf{n}^{l} \mid G_{0} \cap G_{J_{t}} \neq 1\right\}$. Since $G$ is not nilpotent, $b_{l} \geqslant 1$ for all $l$. Now $G_{0} \pi_{J_{t}} \leqslant G_{0} \pi_{J_{l}, 1}$ $\times \cdots \times G_{0} \pi_{J, n}$ implies that $b_{l} \leqslant b_{l+1}$ for all $l$. Hence there exists some positive integer $s_{1}$ such that $b_{s_{1}}=b_{j}$ for all $j \geqslant s_{1}$, because $G_{0}$ is a group with finite composition series. (Actually, it can easily be shown that $b_{s_{1}}=b_{j}$ for all $j \geqslant s_{1}$ is equivalent to $b_{s_{1}}=b_{s_{1}+1}$.) We choose $s_{1}$ minimal with respect to this property and set $b=b_{s_{1}}$. We call $b$ the branching number of the iterated embedding. (This notation is justified by the following fact: as mentioned above, $\mathscr{B}_{l} \subseteq\left\{J_{l} \in \mathbf{n}^{l} \mid\right.$ $\left.G_{0} \cap G_{J_{I}} \neq 1\right\}$. If $G$ does not admit a nontrivial homomorphism into its center (the case we are mainly interested in), then we actually have $\mathscr{B}_{l}=\left\{J_{l} \in \mathbf{n}^{\prime} \mid G_{0} \cap G_{J_{l}}\right.$ $\neq 1$ \} by $3.3(\mathrm{e})$.)

(c) For $k \geqslant s_{1}$, let $\mathscr{B}_{k}=\left\{J_{k}^{1}, \ldots, J_{k}^{b}\right\}$. For $i=1, \ldots, b$, let $J_{s_{1}}^{i}=\left(j_{1}^{i}, \ldots, j_{s_{1}}^{i}\right)$. Since $b_{s_{1}}=b_{s_{1}+1}$, and since $G_{0} \pi_{J_{s_{1}}} \leqslant G_{0} \pi_{J_{s_{1}, 1}^{i}} \times \cdots \times G_{0} \pi_{J_{s_{1, n}}^{i}}$, there exists exactly one index $j_{s_{1}+1}^{i} \in\{1, \ldots, n\}$ such that $J_{s_{1}+1}^{i}=\left(j_{1}^{i}, \ldots, j_{s_{1}+1}^{i}\right)$. Analogously, $j_{s_{1}+2}^{i}$, $j_{s_{1}+3}^{i}, \ldots$ are defined for $i=1, \ldots, b$. For $i=1, \ldots, b$, we denote by $J^{i}$ the sequence $\left(j_{k}^{i} \mid k \in \mathbb{N}\right)$. Let $\mathscr{B}_{\infty}=\left\{J^{i} \mid i=1, \ldots, b\right\}$. Finally, for $1 \leqslant l \leqslant k$ and $i=1, \ldots, b$, set $J_{[l, k]}^{i}=\left(j_{l}^{i}, \ldots, j_{k}^{i}\right)$; thus $J_{k}^{i}=J_{[1, k]}^{i}$.

(d) Let $k \in \mathbb{N}$ and let $i \in\{1, \ldots, b\}$. Then $G_{0} \pi_{J_{k+1}^{i}}=\left(G_{0} \pi_{J_{k}^{i}}\right) \pi_{J_{k+1}^{i}}$ is a homomorphic image of $G_{0} \pi_{J_{k}}$. Application of the maximal condition on normal subgroups to the ascending chain $\operatorname{ker} \pi_{J_{k}^{i}} \cap G_{0}$ yields the existence of some (smallest possible) index $s(i)$ with $G_{0} \pi_{J_{s(i)}^{i}} \cong G_{0} \pi_{J_{j}}$ for all $j \geqslant s(i)$. We set $s=\max \{s(i) \mid i=1, \ldots, b\}$ and call $s$ the stationary level of the iterated embedding. We show in 5.3 that $s \geqslant s_{1}$.

(e) For $k \geqslant s$, define $\mathscr{B}_{k}^{*}=\left\{J_{k}^{i} \in \mathscr{B}_{k} \mid G_{0} \pi_{J_{k}^{j}} \cong G_{0} \pi_{J_{[k+1, k+s]}^{i}}\right\}$ and $\mathscr{B}_{k}^{* *}=\left\{J_{k}^{i}\right.$ $\in \mathscr{B}_{k} \mid G_{0} \pi_{J_{k}}$ is not isomorphic to a proper subnormal subgroup of any $G_{0} \pi_{J_{k}^{j}}$, $j=1, \ldots, b\}$. It will be shown in 5.7 that $J_{k}^{i} \in \mathscr{B}_{k}^{* *}$ for some $k \geqslant s$ if and only if $J_{l}^{i} \in \mathscr{B}_{l}^{* *}$ for all $l \geqslant s$. Therefore, it makes sense to define $\mathscr{B}_{\infty}^{* *}=\left\{J^{i} \in \mathscr{B}_{\infty} \mid J_{k}^{i}\right.$ $\in \mathscr{B}_{k}^{* *}$ for all $\left.k \geqslant s\right\}$. We have been unable to decide whether an analogous result holds for $\mathscr{B}_{k}^{*}$. 
(f) For $k \geqslant s$ and for $i \in\{1, \ldots, b\}$, define the normal subgroup $N_{k}^{i}$ of $G_{J_{k}^{i}}$ by $N_{k}^{i}=\operatorname{ker} \pi_{J_{k+s}^{i}} \cap G_{J_{k}^{i}}=G_{J_{k}^{i}} \cap \times\left\{G_{J_{k}^{i}, J} \mid J \in \mathbf{n}^{s}, J \neq J_{[k+1, k+s]}^{i}\right\}$. It follows from (d) that $N_{k}^{i}=\operatorname{ker} \pi_{J_{k+1}^{i}} \cap G_{J_{k}^{i}}=G_{J_{k}^{i}} \cap \times\left\{G_{J_{k}^{i}, J} \mid J \in \mathbf{n}^{l}, J \neq J_{[k+1, k+l]}^{i}\right\}$ for all $l \geqslant s$.

\subsection{LEMMA. $s \geqslant s_{1}$.}

Proof. Suppose that the assertion is false. Then, by definition of $s$ and $s_{1}$, there exist $h, i \in\{1, \ldots, b\}, h \neq i$, such that $J_{k}^{i}=J_{k}^{h}$ for some $k \geqslant s$, but such that $j_{k+1}^{i} \neq j_{k+1}^{h}$. Since $G_{0} \pi_{J_{k}^{i}} \cong G_{0} \pi_{J_{k+1}^{i}}$, it follows from the maximal condition on normal subgroups in $G_{0} \pi_{J_{k}^{i}}$ that $G_{0} \pi_{J_{k}^{i}} \cap \times_{j \neq j_{k+1}^{i}} G_{J_{k}^{i}, j}=1$. Analogously, $G_{0} \pi_{J_{k}^{i}}$ $\cap \times_{j \neq j_{k+1}^{h}} G_{J_{k}^{i}, j}=1$. A fortiori, $G_{0} \cap G_{J_{k}^{i}, j}=1$ for all $j=1, \ldots, n$. By 3.3(e), this implies that $G_{0} \pi_{J_{k+1}^{i}}$ and $G_{0} \pi_{J_{k+1}^{h}}$ are nilpotent, contradicting the fact that $J_{k+1}^{i}, J_{k+1}^{h} \in \mathscr{B}_{k+1}$.

5.4. Lemma. For $i \in\{1, \ldots, b\}$ and for $m \geqslant 1$, the sequence $\left(j_{i}^{i} \mid l \geqslant m\right)$ is $a$ member of $\mathscr{B}_{\infty}$. Moreover, $G_{0} \pi_{J_{s(i)}^{i}}$ is isomorphic to a subnormal subgroup of $G_{0} \pi_{J_{[m, m+r]}^{i}}$ for any $r \geqslant s(i)-1$.

Proof. Let $r \geqslant s(i)-1$. We have $G_{0} \pi_{J_{s(i)}^{i}} \cong G_{0} \pi_{J_{m+r}^{i}}$ as $m+r \geqslant s(i)$. Since $J_{s(i)}^{i} \in \mathscr{B}_{s(i)}$ and $G_{0} \pi_{J_{m+r}^{i}} \unlhd \unlhd G_{J_{m-1}^{i}} \pi_{J_{m+r}^{i}} \cong G_{0} \pi_{J_{[m, m+r]}^{i}}$, we conclude that $G_{0} \pi_{J_{[m, m+r]}^{i}}$ is not nilpotent. The assertion follows.

5.5. Lemma. Let $i \in\{1, \ldots, b\}$ and let $k \geqslant s$.

(a) The following statements are equivalent:

(i) $J_{k}^{i} \in \mathscr{B}_{k}^{*}$;

(ii) $G_{0} \pi_{J_{k}^{i}} \cong G_{0} \pi_{J_{[k+1, k+1]}^{i}}$ for some $l \geqslant s$;

(iii) $G_{0} \pi_{J_{k}^{i}} \cong G_{0} \pi_{J_{[k+1, k+l]}^{i}}$ for all $l \geqslant s$;

(iv) $J_{k}^{i} \in \mathscr{B}_{k}$, and $N_{k}^{i}$ is complemented by $G_{0} \pi_{J_{k}}$ in $G_{J_{k}^{i}}$.

(b) Let $1 \leqslant m \leqslant k+1$ and let $s \leqslant l \leqslant k$. If $J_{k}^{i} \in \mathscr{B}_{k}^{*}$, then $J_{l}^{i} \in \mathscr{B}_{l}^{*}$. Moreover, $G_{0} \pi_{J_{s}^{i}} \cong G_{0} \pi_{J^{i}, m+s-1 \mid}$

(c) Let $J_{k}^{i} \stackrel{m, m \mathscr{B}_{k}^{*}}{\in}$ be such that $J_{2 k}^{i}=\left(J_{k}^{i}, J_{k}^{i}\right)$. Then $J_{m k}^{i}=\left(J_{(m-1) k}^{i}, J_{k}^{i}\right)$ for all $m \geqslant 2$, and $J_{i}^{i} \in \mathscr{B}_{i}^{*}$ for all $l \geqslant s$. Moreover, if $\sigma$ denotes the $k$-cycle $(1, \ldots, k)$ in the symmetric group $S_{k}$, then $J_{k}^{i} \sigma^{r} \in \mathscr{B}_{k}^{*}$ and $G_{0} \pi_{J_{k}^{i}} \cong G_{0} \pi_{J_{k}^{i} \sigma^{r}} \cong G_{0} \pi_{\left(J_{k}^{i} \sigma^{r}, J_{k}^{i} \sigma^{r}\right)}$ for all $r$.

Proof. (a) It follows from 5.4 that (i), (ii), and (iii) are equivalent. Suppose $J_{k}^{i} \in \mathscr{B}_{k}^{*}$. Then $G_{0} \pi_{J_{k}} /\left(N_{k}^{i} \cap G_{0} \pi_{J_{k}}\right) \cong G_{0} \pi_{J_{k+s}^{i}} \cong G_{0} \pi_{J_{k}^{j}}$. Now $G_{0} \pi_{J_{k}}$, being an epimorphic image of $G_{0}$, satisfies the maximal condition on normal subgroups. 
Hence, $N_{k}^{i} \cap G_{0} \pi_{J_{k}^{j}}=1$. Furthermore, $G_{J_{k}^{i}} / N_{k}^{i} \cong G_{J_{k}^{i}} \pi_{J_{k+s}^{i}} \cong G_{0} \pi_{J_{|k+1, k+s|}^{i}} \cong G_{0} \pi_{J_{k}^{i}}$, as $J_{k}^{i} \in \mathscr{B}_{k}^{*}$. Since $G_{J_{k}^{i}}$ satisfies the minimal condition on subnormal subgroups, (iv) follows.

If (iv) holds, then $G_{0} \pi_{J_{k}^{i}} \cong G_{J_{k}} / N_{k}^{i} \cong G_{J_{k}^{i}} \pi_{J_{k+s}^{i}} \cong G_{0} \pi_{J_{i k+1, k+s}}$; thus $J_{k}^{i} \in \mathscr{B}_{k}^{*}$.

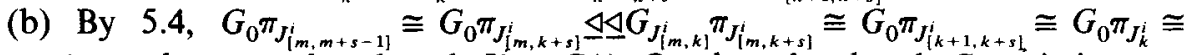
$G_{0} \pi_{J_{s}^{i}}$ (note that $m \leqslant k+1$ and $J_{k}^{i} \in \mathscr{B}_{k}^{*}$ ). On the other hand, $G_{0} \pi_{J_{s}^{i}}$ is isomorphic to a subnormal subgroup of $G_{0} \pi_{J_{[m, m+s-1]}^{i}}$ by 5.4. The minimal condition on subnormal subgroups implies that $G_{0} \pi_{J^{i m, m+s-1]}} \cong G_{0} \pi_{J_{s}^{i}}$. Setting $m=l+1 \leqslant$ $k+1$, we obtain $G_{0} \pi_{J^{i+1, i+s \mid}} \cong G_{0} \pi_{J_{s}^{i}} \cong G_{0} \pi_{J_{i}}$. Hence $J_{l}^{i} \in \mathscr{B}_{l}^{*}$.

(c) We prove $J_{m k}^{i}=\left(J_{(m-1) k}^{i}, J_{k}^{i}\right)$ by induction on $m$. The case $m=2$ holds by hypothesis, and so we assume that $m \geqslant 3$. Let $J_{m k}^{i}=\left(J_{(m-1) k}^{i}, J\right), J \in \mathbf{n}^{k}$. By the induction hypothesis, $G_{J_{(m-2) k}^{i}} \pi_{\left(J_{(m-1) k}^{i}, J_{k}^{i}\right)} \cong G_{0} \pi_{J_{2 k}^{i}}$, and this group is not nilpotent. On the other hand, $G_{0} \pi_{J_{m k}^{i}} \unlhd \unlhd G_{J_{(m-2) k}^{i}}^{i} \pi_{J_{m k}^{i}}$. Hence $G_{J_{(m-2) k}^{i}} \pi_{J_{m k}^{i}}=G_{J_{(m-2) k}^{i}} \pi_{\left(J_{(m-1) k}^{i}, J\right)}$ is not nilpotent. Since $k \geqslant s$, we conclude that $J=J_{k}^{i}$.

Let $l \geqslant s$ be arbitrary and choose $m$ such that $m k \geqslant l$. Since $J_{m k}^{i}=J_{[m k+1,2 m k]}^{i}$, part (a) shows that $J_{m k}^{i} \in \mathscr{B}_{m k}^{*}$. By part (b), $J_{l}^{i} \in \mathscr{B}_{l}{ }^{*}$. That $G_{0} \pi_{J_{k}} \cong G_{0} \pi_{J_{k}^{i} \sigma^{r}} \cong$ $G_{0} \pi_{\left(J_{k}^{i} \sigma^{r}, J_{k}^{i} \sigma^{r}\right)}$ holds follows from the preceding arguments, from part (b), and from 5.4. In particular, $J_{k}^{i} \sigma^{r} \in \mathscr{B}_{k}^{*}$.

5.6. Lemma. Let $k \geqslant s$ and $i \in\{1, \ldots, b\}$ be such that $J_{k}^{i} \in \mathscr{B}_{k}^{*}$. Then

(a) $\left(G_{0} \pi_{J_{k}^{j}}\right)_{J_{k}} /$ Core $_{G_{j k}}\left(G_{0} \pi_{J_{k}^{j}}\right)$ is nilpotent,

(b) $\left[G_{0} \pi_{J_{k}}, N_{k}^{i}\right] \leqslant \operatorname{Fit}\left(G_{J_{k}^{i}}\right)$.

Proof. Recall that, by 5.5(a), $G_{0} \pi_{J_{k}^{i}}$ is a complement to $N_{k}^{i}$ in $G_{J_{k}^{i}}$.

(a) Clearly, $G_{0} \cap G_{J_{k+s}^{i}} \unlhd G_{0} \pi_{J_{k}}$. By 3.3(a), $G_{0} \pi_{J_{k}} /\left(G_{0} \cap G_{J_{k+s}^{t}}\right)$ is nilpotent. Moreover, $\left[N_{k}^{i}, G_{0} \cap G_{J_{k+s}^{i}}\right] \leqslant N_{k}^{i} \cap G_{J_{k+s}^{i}}=1$ according to the definition of $N_{k}^{i}$. Thus $G_{0} \cap G_{J_{k+s}^{\prime}} \unlhd G_{J_{k^{i}}}$, and $G_{0} \pi_{J_{k}} / \operatorname{Core}_{G_{J_{k}^{\prime}}}\left(G_{0} \pi_{J_{k}^{\prime}}\right)$ is nilpotent. Since $G_{0} \pi_{J_{k}^{i}}$ is subnormal in $G_{J_{k}}$, the conclusion follows from 3.1(a).

(b) This is clear from (a).

5.7. Lemma. Let $i \in\{1, \ldots, b\}$. If $J_{k}^{i} \in \mathscr{B}_{k}^{* *}$ for some $k \geqslant s$, then $J_{l}^{i} \in \mathscr{B}_{l}^{* *}$ for all $l \geqslant s$. Moreover, if $m \geqslant 1$, then $G_{0} \pi_{J_{l m, m+s-1)}^{i}} \cong G_{0} \pi_{J_{s}}$; in particular, $J_{[m, m+s-1]}^{i} \in \mathscr{B}_{s}^{* *}$.

Proof. This follows immediately from the definition and from 5.4.

\subsection{LemMA. Let $k \geqslant s$. Then $\varnothing \neq \mathscr{B}_{k}^{* *} \subseteq \mathscr{B}_{k}^{*}$.}

Proof. Since $G$ is not nilpotent, $\mathscr{B}_{k} \neq \varnothing$. Therefore, $\mathscr{B}_{k}^{* *} \neq \varnothing$ by virtue of the minimal condition on subnormal subgroups. Now let $J_{k}^{i} \in \mathscr{B}_{k}^{* *}$. As in the proof of the implication (i) $\Rightarrow$ (iv) in 5.5(a) it follows that $G_{0} \pi_{J_{k}^{i}} \cap N_{k}^{i}=1$. Hence 
$G_{0} \pi_{J_{k}^{i}} \cong\left(G_{0} \pi_{J_{k}}\right) N_{k}^{i} / N_{k}^{i} \unlhd \unlhd G_{J_{k}^{i}} / N_{k}^{i} \cong G_{J_{k}} \pi_{J_{k+s}^{i}} \cong G_{0} \pi_{J_{[k+1, k+s]}^{i}}$. From $J_{k}^{i} \in \mathscr{B}_{k}^{* *}$ we conclude that $G_{0} \pi_{J_{k}^{i}} \cong G_{0} \pi_{\left.J_{[k+1, k+s]}^{i}\right]}$, i.e. that $J_{k}^{i} \in \mathscr{D}_{k}^{*}$.

5.9. Lemma. Let $i \in\{1, \ldots, b\}$ be such that $J_{s}^{i} \in \mathscr{B}_{s}^{* *}$. Then there exists $l \geqslant s$ and $J_{l}^{h} \in \mathscr{B}_{l}^{* *}($ for some $h \in\{1, \ldots, b\})$ with $J_{[l+1,2 l]}^{h}=J_{l}^{h}$ and $G_{0} \pi_{J_{s}^{i}} \cong G_{0} \pi_{J_{l}^{h}}$.

Proof. It follows from 5.7 that $J_{[u s+1,(u+1) s]}^{i} \in \mathscr{B}_{s}^{* *}$ for all positive integers $u$. Since $\mathscr{B}_{s}^{* *}$ is a finite set, there exist positive integers $a<b$ such that $J_{[a s+1,(a+1) s]}^{i}=J_{[b s+1,(b+1) s]}^{i}$. Set $l=s(b-a) \geqslant s$ and $J=J_{[a s+1, b s]}^{i} \in \mathbf{n}^{l}$. Then $G_{0} \pi_{J_{s}^{i}} \cong G_{0} \pi_{J_{\text {os }}^{i}} \cong G_{0} \pi_{J}$ by 5.7. Hence, $J \in \mathscr{B}_{l}^{* *}$, say $J=J_{l}^{h}$ for some $h \in$ $\{1, \ldots, b\}$. It remains to show that $J_{l}^{h}=J_{[l+1,2 l]}^{h}$. Using 5.4, we infer from $J_{[a s+1,(a+1) s]}^{i}=J_{[b s+1,(b+1) s]}^{i}$ that $G_{0} \pi_{J_{l}^{h}}=G_{0} \pi_{J_{[a s+1, b s]}^{l}} \cong G_{0} \pi_{J_{[a s+1,(b+1) s]}^{i}}=$ $G_{0} \pi_{\left(I_{l}^{h}, J_{[a s+1,(a+1) s)}^{i}\right)}$. This proves the assertion in the case $b=a+1$. So we may assume that $b>a+1$. Now $G_{0} \pi_{\left(J_{[a s+1,(a+1) s]}^{i}, I\right)}$ is nilpotent for all $I \in \mathbf{n}^{(s-1)(b-a)}$, $I \neq J_{[(a+1) s+1, b s]}^{i}$, because $J_{[a s+1,(a+1) s]}^{i} \in \mathscr{B}_{s}$ and $G_{0} \pi_{\left(J_{[a s+1,(a+1) s]}^{i}, J_{[(a+1) s+1, b s]}^{i}\right)}=$ $G_{0} \pi_{J_{l}^{h}}$ is not nilpotent. Since $G_{0} \pi_{\left(J_{l}^{h}, J_{l a s+1,(a+1) s]}, I\right)} \leqslant G_{J_{l}^{h}} \pi_{\left(J_{l}^{h}, J_{[a s+1,(a+1) s]}^{i}, I\right)} \cong$ $G_{0} \pi_{\left(J_{[a s+1,(a+1) s]}^{l}, I\right)}$ for all $I \in \mathbf{n}^{(s-1)(b-a)}$, we conclude that $G_{0} \pi_{\left(J_{l}^{h}, J_{(a s+1,(a+1) s}^{l}, I\right)}$ is nilpotent for all $I \in \mathbf{n}^{(s-1)(b-a)}, I \neq J_{[(a+1) s+1, b s]}^{i}$. It follows that $J_{[l+1,2 l]}^{h}=J_{l}^{h}$.

The following theorem rests upon the results obtained so far in this section. It exhibits a particular nice factorization and implies the validity of Theorem 5.1 (note the remarks following the statement of 5.1).

5.10. THEOREM. Let $G$ be a group with finite composition series. Let $G_{0} \unlhd \unlhd$ $G_{1} \times \cdots \times G_{n}$ be a non-trivial subnormal embedding (cf. 3.4), where $G_{j} \cong G$ for all $j=0,1, \ldots, n$. Then either $G$ is nilpotent or the following statements hold (with the notation of 5.2(a)). There exists some positive integer $l$ and $J_{l}=\left(j_{1}, \ldots, j_{l}\right) \in \mathbf{n}^{l}$ such that

(a) $G_{J_{l}}=N_{J_{l}} \cdot G_{0} \pi_{J}$, where $N_{J_{l}}=G_{J_{l}} \cap \times\left\{G_{J_{l}, J} \mid J \in \mathbf{n}^{l}, J \neq J_{l}\right\}$

(b) $N_{J_{t}} \cap G_{0} \pi_{J_{t}}=1$

(c) $G_{0} \pi_{J_{l}}$ is a non-nilpotent subnormal subgroup of $G_{J_{l}}$

(d) $N_{J_{I}}$ is a non-trivial normal subgroup of $G_{J_{I}}$

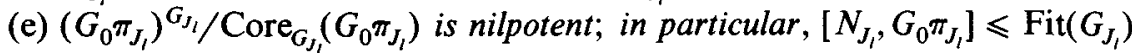

(f) $G_{0} \cap G_{J_{t}}=G_{J_{I}} \cap G_{J_{I}, J_{I}} \unlhd G_{J_{i}}$

(g) $G_{J_{m}}=\left(G_{J_{m}} \cap \times\left\{G_{J_{m}, J} \mid J \in \mathbf{n}^{l}, J \neq J_{l} \sigma^{m}\right\}\right) \cdot G_{0} \pi_{J_{m}}$ for $1 \leqslant m \leqslant l$, where $J_{m}=\left(j_{1}, \ldots, j_{m}\right)$ and $\sigma=(1, \ldots, l) \in S_{l}$.

Proof. Let $G$ be non-nilpotent. According to 5.2 , let $\mathscr{B}_{k}=\left\{J_{k}^{1}, \ldots, J_{k}^{b}\right\}$ for $k \geqslant s$. Now $G_{0}$ satisfies the minimal condition on normal subgroups. Since $G_{0} \cap G_{J_{k}} \geqslant G_{0} \cap G_{J_{k+1}}$ for all $k \geqslant s$ and all $i=1, \ldots, b$, we deduce the existence 
of some integer $t \geqslant s$ such that

$$
G_{0} \cap G_{J_{k}^{i}}=G_{0} \cap G_{J_{k+1}^{i}} \text { for all } k \geqslant t \text { and for } i=1, \ldots, b .
$$

Since, by 5.8, $\mathscr{B}_{s}^{* *} \neq \varnothing$, we can choose $l$ and $J_{l}^{h} \in \mathscr{B}_{l}^{* *}$ as in 5.9. By 5.5(c), we may replace $l$ by al $(a \in \mathbb{N})$ without violating the statement of 5.9. Hence, without loss of generality, we have $l \geqslant t$.

Set $J_{l}=J_{l}^{h}$. Then (a) and (b) follow immediately from 5.8 and from 5.5(a). Statement (c) is a consequence of $J_{l} \in \mathscr{B}_{l}^{* *} \subseteq \mathscr{B}_{l}$. The fact that the embedding of $G_{J_{l}}$ in $G_{J_{l}, 1} \times \cdots \times G_{J_{l}, n}$ is non-trivial yields (d). Part (e) is proved in 5.6.

By the choice of $J_{l}$, and because of (*), we have $G_{0} \cap G_{J_{l}}=G_{0} \cap G_{J_{l}, J_{i}}$. Thus $G_{0} \cap G_{J_{l}} \unlhd \unlhd G_{J_{I}} \cap G_{J_{l}, J_{l}} \cong G_{0} \cap G_{J_{l}}$. Hence the minimal condition on subnormal subgroups implies the validity of statement (f).

Finally, let $1 \leqslant m \leqslant l$. Set $N_{J_{m}}=G_{J_{m}} \cap \times\left\{G_{J_{m}, J} \mid J \in \mathbf{n}^{l}, J \neq J_{l} \boldsymbol{\sigma}^{m}\right\}$. Then $G_{0} \pi_{J_{m}} /\left(G_{0} \pi_{J_{m}} \cap N_{J_{m}}\right) \cong G_{0} \pi_{\left(J_{m}, J_{l} \sigma^{m}\right)}=G_{0} \pi_{\left(J_{l}, J_{m}\right)} \cong G_{0} \pi_{J_{l}}$. Furthermore, $G_{J_{m}} / N_{J_{m}}$ $\cong G_{J_{m}} \pi_{\left(J_{m}, J_{l} \sigma^{m}\right)} \cong G_{0} \pi_{J_{l} \sigma^{m}} \cong G_{0} \pi_{J_{l}}$, where the latter isomorphism is given by $5.5(\mathrm{c})$. Employing once again the minimal condition on subnormal subgroups in $G_{J_{m}}$, we obtain $(\mathrm{g})$.

5.11. REMARK. Let $G$ be a finite non-nilpotent group satisfying the hypotheses of Theorem 5.10. Assume further that, for every prime divisor $p$ of $\left|G / G^{\prime}\right|$, the Sylow $p$-subgroups of $G$ are abelian. Then $G$ is directly decomposable (in a non-trivial way). In view of 5.10 , this follows from the following simple fact: if the finite group $G$ has abelian Sylow $p$-subgroups for every prime divisor $p$ of $\left|G / G^{\prime}\right|$, and if $G$ is of the form $G=N S$, where $N \unlhd G, S \unlhd \unlhd G, N \cap S=1$, and $S /$ Core $_{G}(S)$ is nilpotent, then $G=N \times \tilde{S}$ for some $\tilde{S} \leqslant G$. This is proved by induction on $|G|$. By standard reductions, we may assume that $\operatorname{Core}_{G}(S)=1$ and that $S \leqslant O_{p}(G)$ for some prime $p \cdot\left(O_{p}(G)\right.$ denotes the largest normal $p$-subgroup of $G$.) Define $T=[S, N] S \leqslant O_{p}(G)$. Then $T$ is $N$-invariant. Since $p$ divides $\left|G / G^{\prime}\right|$, the Sylow $p$-subgroups of $G$ are abelian. Consequently, $T$ is abelian, and $p$ does not divide $\left|N / C_{N}(T)\right|$. Therefore, $T=[T, N] \times C_{T}(N)$ [4, III.13.4(b)]. Let $S=\left\langle s_{1}, \ldots, s_{m}\right\rangle$. There exist $x_{i} \in[T, N]$ such that $s_{i} x_{i} \in C_{T}(N)$ for $i=$ $1, \ldots, m$. Define $\tilde{S}=\left\langle s_{1} x_{1}, \ldots, s_{m} x_{m}\right\rangle \leqslant C_{T}(N)$. Since $[T, N] \leqslant N$, we conclude that $G=N S=N \tilde{S}$. Let $y=\prod_{i=1}^{m}\left(s_{i} x_{i}\right)^{a_{i}}=\prod_{i=1}^{m} s_{i}^{a_{i}} \prod_{i=1}^{m} x_{i}^{a_{i}} \in N \cap \tilde{S}$, where $a_{i} \in \mathbf{Z}$ (note that $T$ is abelian). Then $\prod_{i=1}^{m} s_{i}^{a_{i}} \in N \cap S=1$; hence $y=\prod_{i=1}^{m} x_{i}^{a_{i}}$ $\in[T, N] \cap \tilde{S} \leqslant[T, N] \cap C_{T}(N)=1$. Consequently, $\tilde{S}$ is a complement for $N$ in $G$. Clearly, then, $G=N \times \tilde{S}$, as $\tilde{S} \leqslant C_{T}(N)$.

5.12. Examples. (a) (J. Cossey) The following example exhibits a directly indecomposable finite group $G$ with trivial center which possesses a non-trivial subnormal embedding in $G \times G$ (in the sense of 3.4 ). 
Let $G$ be generated by $x_{1}, x_{2}, x_{3}, y_{1}, y_{2}$ according to the following defining relations:

$$
\begin{gathered}
x_{1}^{3}=x_{2}^{3}=x_{3}^{3}=\left[x_{1}, x_{3}\right]^{3}=\left[x_{2}, x_{3}\right]^{3}=y_{1}^{2}=y_{2}^{2}=1, \\
{\left[x_{1}, x_{2}\right]=1, \quad\left[x_{1}, x_{3}, x_{i}\right]=\left[x_{2}, x_{3}, x_{i}\right]=1 \text { for } i=1,2,3,} \\
{\left[x_{2}, y_{1}\right]=\left[x_{3}, y_{1}\right]=\left[x_{1}, y_{2}\right]=\left[x_{3}, y_{2}\right]=\left[y_{1}, y_{2}\right]=1,} \\
{\left[x_{1}, y_{1}\right]=x_{1}, \quad\left[x_{2}, y_{2}\right]=x_{2} .}
\end{gathered}
$$

Then $|G|=2^{2} 3^{5}, Z(G)=1$, and $G$ is directly indecomposable. (If $V$ denotes the indecomposable faithful $Z_{6}$-module over $G F(3)$ of dimension 2 and $H$ the semidirect product of $V$ with $Z_{6}$, then $G$ is the direct product of two copies of $H$ with amalgamated factor group $Z_{3}$.)

Let $G^{*}$ be a copy of $G$ with generators $x_{i}^{*}, y_{j}^{*}$ that correspond to $x_{i}, y_{j}$. It is easy to check that $G_{0}=\left\langle x_{1}, x_{1}^{*}, x_{3} x_{3}^{*}, y_{1}, y_{1}^{*}\right\rangle$ is a subnormal subgroup of $G \times G^{*}$ which is isomorphic to $G$ ( $x_{1}$ corresponds to $x_{1}, x_{1}^{*}$ to $x_{2}, x_{3} x_{3}^{*}$ to $x_{3}$, $y_{1}$ to $y_{1}$, and $y_{1}^{*}$ to $\left.y_{2}\right)$. Clearly, $G_{0} \cap G \neq 1 \neq G_{0} \cap G^{*}$.

(b) Groups $G$ with the structural properties described in 5.1(c) need not admit non-trivial subnormal embeddings in direct products of groups isomorphic to $G$.

Let $P=\left\langle x, y \mid x^{3}=y^{3}=[x, y, x]=[x, y, y]=1\right\rangle$ be an extraspecial group of order 27 and exponent 3, and let $z$ denote the automorphism of $P$ which inverts $x$ (and $[x, y]$ ) and leaves $y$ invariant. Let $R$ be the semidirect product of $P$ and $\langle z\rangle$. Then $N=\langle x,[x, y], z\rangle$ is a normal subgroup of index 3 in $R$. Moreover, $R$ acts on a cyclic group $\langle t\rangle$ of order 7 with kernel $N$, and $[t, y]=t$. Finally, let $G$ be the semidirect product of $\langle t\rangle$ and $R$ with respect to this action. Then $Z(G)=1$. Since $\langle t\rangle$ and $\langle[x, y]\rangle$ are the only minimal normal subgroups of $G$, it is easy to verify that $G$ does not allow non-trivial subnormal embeddings in direct products of groups isomorphic to $G$. However, statement 5.1(c) is satisfied with $N$ as above and with $S=\langle y, t\rangle$.

It remains an open question as to how those groups $G$ can be characterized which admit a non-trivial subnormal embedding in a direct product of groups isomorphic to $G$.

We conclude this section with a result on the subnormal embeddings of a group which does not admit a non-trivial homomorphism into its center. This will be of use in our investigations on normally detectable groups.

5.13. Proposition. Let $G$ be a group satisfying the maximal condition on subnormal subgroups which does not admit a non-trivial homomorphism into its center. Let $G_{0} \unlhd \unlhd G_{1} \times \cdots \times G_{n}$, where $G_{j} \cong G$ for $j=0,1, \ldots, n$. If $N$ is a minimal normal subgroup of $G_{0}$, and if $i \in\{1, \ldots, n\}$, then either $N \pi_{i}=1$, or $N \pi_{i}$ is a minimal normal subgroup of $G_{0}$. 
Proof. Suppose that $N \pi_{i} \neq 1$. Then, if $1 \neq M_{i} \leqslant N \pi_{i}$ is a normal subgroup of $G_{0} \pi_{i}$, it follows that $1 \neq M=\left\{n \in N \mid n \pi_{i} \in M_{i}\right\}$ is a normal subgroup of $G_{0}$. Hence $N \pi_{i}$ is a minimal normal subgroup of $G_{0} \pi_{i}$. Aiming for a contradiction, we assume that $N \pi_{i}$ is not a minimal normal subgroup of $G_{0}$. Then $N \pi_{i} \nless G_{0}$. Moreover, $N \cap \times_{j=1}^{n}\left(G_{0} \cap G_{j}\right)=1$; for otherwise, $N \leqslant \times_{j=1}^{n}\left(G_{0} \cap G_{j}\right)$, and hence $N \pi_{i} \leqslant G_{0} \cap G_{i} \leqslant G_{0}$, a contradiction.

Now $\left[N \pi_{i} \cap G_{0}, G_{0} \pi_{i}\right]=\left[N \pi_{i} \cap G_{0}, G_{0}\right] \leqslant N \pi_{i} \cap G_{0}$. Therefore, $N \pi_{i} \cap$ $G_{0} \unlhd G_{0} \pi_{i}$. Since $N \pi_{i} \nless G_{0}$ is a minimal normal subgroup of $G_{0} \pi_{i}$, we conclude that $N \pi_{i} \cap G_{0}=1$. By $3.3(\mathrm{a}), G_{0} \pi_{i} /\left(G_{0} \cap G_{i}\right)$ is nilpotent, say of class $c$. Then [ $N \pi_{i}$, $\left.G_{0} \pi_{i}, \ldots, G_{0} \pi_{i}\right] \leqslant N \pi_{i} \cap G_{0} \cap G_{i}=1$ (here $G_{0} \pi_{i}$ occurs $c$ times). Hence $N \pi_{i}$ is contained in the hypercenter and, as a minimal normal subgroup, even in the center of $G_{0} \pi_{i}$. Consequently, $\left[N, G_{0}\right] \leqslant N \cap \times_{j \neq i} G_{j}$. Since $N \pi_{i} \neq 1$ and $N \cap \times_{j \neq i} G_{j} \unlhd G_{0}, N$ is contained in $Z\left(G_{0}\right)$. By 3.3(a) again, $G_{0} / \times_{j=1}^{n}\left(G_{0} \cap G_{j}\right)$ is nilpotent. Moreover, $N \cong N\left(\times_{j=1}^{n}\left(G_{0} \cap G_{j}\right)\right) /\left(\times_{j=1}^{n}\left(G_{0} \cap G_{j}\right)\right) \leqslant$ $Z\left(G_{0} / \times_{j=1}^{n}\left(G_{0} \cap G_{j}\right)\right)$; this holds because $N \cap \times_{j=1}^{n}\left(G_{0} \cap G_{j}\right)=1$ and $N \leqslant$ $Z\left(G_{0}\right)$. As a nilpotent factor group of $G_{0}, G_{0} / \times_{j=1}^{n}\left(G_{0} \cap G_{j}\right)$ is finitely generated. By means of 3.2 we deduce the existence of a non-trivial homomorphism from $G_{0} / \times_{j=1}^{n}\left(G_{0} \cap G_{j}\right)$ into $N \leqslant Z\left(G_{0}\right)$, the desired contradiction.

\section{Normally detectable groups}

6.1. Definition. A group $G$ is called normally detectable if the following holds: whenever $G_{0}$ is normal in $G_{1} \times \cdots \times G_{n}$ for some positive integer $n$, where $G_{j} \cong G$ for $j=0,1, \ldots, n$, then $G_{0}=G_{i}$ for some $i \in\{1, \ldots, n\}$.

The following conjecture is due to T. O. Hawkes.

CONJECTURE. A finite group $G$ is normally detectable if and only if

(1) $G$ is directly indecomposable, and

(2) $\left(\left|G / G^{\prime}\right|,|Z(G)|\right)=1$.

It is clear that (1) and (2) are necessary for a group to be normally detectable: for (1), the same argument as in the proof of the implication (i) $\Rightarrow$ (ii) of Theorem 4.2 works. For (2), assume that there is a non-trivial homomorphism $\alpha: G \rightarrow Z(G)$. Then $G_{0}=\{(g, g \alpha) \mid g \in G\}$ is a normal subgroup of $G \times G, G_{0} \cong G$.

We are not able either to prove or disprove Hawkes' conjecture. This section is devoted to the proofs of several partial results. We show that if one of the conditions (1) or (2) is somewhat strengthened, it follows that the group in question is normally detectable (Theorems 6.2 and 6.5). Moreover, for various 
types of groups we are able to prove that Hawkes' conjecture holds (Theorem 6.9).

To obtain these results, we not only need information about the structure but also about the type of embedding of a possible counterexample (Theorem 6.8). Theorem 5.1 already shows that a group which is a counterexample to Hawkes' conjecture has to admit a certain factorization. In fact, in dealing with normal embeddings we can describe this factorization more precisely than for general subnormal embeddings.

The following theorem collects this information. Beforehand, we mention that in a group $G$ with finite composition series the center $Z(G)$ and the commutator factor group $G / G^{\prime}$ are finite. Hence for such groups the condition $\left(\left|G / G^{\prime}\right|\right.$, $\mid Z(G) D=1$ is meaningful and equivalent to the non-existence of a non-trivial homomorphism from $G$ into $Z(G)$.

6.2. THEOREM. Let $G$ be a group with finite composition series. Assume that $G$ is directly indecomposable and that $\left(\left|G / G^{\prime}\right|,|Z(G)|\right)=1$. If $G$ is not normally detectable, then the following hold.

(a) $G=N S, N \cap S=1, N \unlhd G, S \unlhd \unlhd G$.

(b) $N$ and $S$ are not nilpotent.

(c) $S^{G} /$ Core $_{G}(S)$ is nilpotent; in particular, $[N, S] \leqslant \operatorname{Fit}(G)$.

(d) $[N, S] \leftleftarrows \operatorname{Soc}(G)$.

(e) $[N, S]$ is not characteristic in $G$; more precisely, there exists an automorphism $\alpha$ of $G$ such that $\langle\alpha\rangle \cong H / M$ for some $H \leqslant G, M \unlhd H$, which does not leave $[N, S]$ invariant.

Proof. We note first that $G \neq 1$ since $G$ is not normally detectable. The condition $\left(\left|G / G^{\prime}\right|,|Z(G)|\right)=1$ then implies that $G$ is not nilpotent. By assumption, there is a non-trivial normal embedding $G_{0} \unlhd G_{1} \times \cdots \times G_{n}$, where $n \geqslant 2$, where $G_{j} \cong G$ for $j=0,1, \ldots, n$, and where $G_{0} \pi_{i} \neq 1$ for $i=1, \ldots, n$. In the sequel we use the notation from 5.2. In particular, $\mathscr{B}_{l}=\left\{J_{l}^{1}, \ldots, J_{l}^{b}\right\}$ for every $l \geqslant s$. By 5.7 and 5.8 we may assume that there is some $a, 1 \leqslant a \leqslant b$, such that $\mathscr{B}_{l}^{* *}=\left\{J_{l}^{1}, \ldots, J_{l}^{a}\right\}$ for all $l \geqslant s$.

Now the proof is carried out in a number of steps.

(1) For all $l \geqslant s$ and all $J_{l}^{i} \in \mathscr{B}_{l}^{* *}$, there exists $J_{s}^{j} \in \mathscr{B}_{s}$ such that $G_{J_{i}^{i}} \cap$ $\left(\operatorname{Soc}\left(G_{J_{i}^{i}}\right) \cap\left[N_{l}^{i}, G_{0} \pi_{J_{i}}\right]\right) \pi_{J_{i}^{i}, J_{s}^{i}} \neq 1$.

$\left[N_{l}^{i}, G_{0} \pi_{J_{i}}\right] \neq 1$ as $G_{J_{i}^{i}}$ is directly indecomposable, and $N_{l}^{i} \neq 1 \neq G_{0} \pi_{J_{i}}$. Since $\left[N_{i}^{i}, G_{0} \pi_{J_{i}}\right] \unlhd N_{l}^{i} \cdot G_{0} \pi_{J_{i}^{i}}=G_{J_{i}}(5.8$ and $5.5(\mathrm{a}))$, there exists a minimal normal subgroup $N$ of $G_{J_{i}^{i}}$ contained in $\left[N_{l}^{i}, G_{0} \pi_{J_{i}}\right]$. Let $J \in \mathbf{n}^{s}$ be such that $N \pi_{J_{i}, J} \neq 1$. By $5.13, N \pi_{J_{i}^{i}, J} \leqslant G_{J_{i}}$. Hence, according to $3.3(\mathrm{e}), J \in \mathscr{B}_{s}$, say $J=J_{s}^{j}$. This proves (1). 
(2) For all $l \geqslant s$ and all $i \in\{1, \ldots, a\}$ (i.e. $J_{l}^{i} \in \mathscr{B}_{l}^{* *}$ ), put

$$
\mathscr{C}\left(J_{l}^{i}\right)=\left\{J_{s}^{j} \in \mathscr{B}_{s} \mid G_{J_{i}} \cap\left(\operatorname{Soc}\left(G_{J_{i}^{i}}\right) \cap\left[N_{i}^{i}, G_{0} \pi_{J_{i}}\right]\right) \pi_{J_{i}, J_{s}^{j}} \neq 1\right\}
$$

and $c\left(J_{l}^{i}\right)=\left|\mathscr{C}\left(J_{l}^{i}\right)\right|$. By (1), $c\left(J_{l}^{i}\right) \geqslant 1$ for all $l \geqslant s$ and for all $i \in\{1, \ldots, a\}$. We choose $t \geqslant s$ and $i \in\{1, \ldots, a\}$ such that $c\left(J_{t}^{i}\right) \leqslant c\left(J_{m}^{j}\right)$ for all $m \geqslant s$ and for all $j \in\{1, \ldots, a\}$. Without loss of generality, we may assume that $i=1$.

(3) There exists some $r \geqslant s$ and $J_{s}^{k} \in \mathscr{B}_{s}$ such that

$$
G_{J_{r+1}^{1}} \cap\left(\operatorname{Soc}\left(G_{J_{r+1}^{1}}\right) \cap\left[N_{r+1}^{1}, G_{0^{\prime}} \pi_{J_{r+1}^{1}}\right]\right) \pi_{J_{r+1}^{1}, J_{s}^{k}} \neq 1
$$

and

$$
G_{J_{r}^{1}} \cap\left(\operatorname{Soc}\left(G_{J_{r}^{1}}\right) \cap\left[N_{r}^{1}, G_{0^{\prime}} \pi_{J_{r}^{1}}\right]\right) \pi_{J_{r+1}^{1}, J_{s}^{k}}=1 .
$$

For $k=1, \ldots, s$, let $\left(\alpha_{t+k}\right)$ denote the following statement:

$$
\left(\alpha_{t+k}\right)\left\{\begin{array}{l}
\text { If } G_{J_{t+k}^{1}} \cap\left(\operatorname{Soc}\left(G_{J_{t+k}^{1}}\right) \cap\left[N_{t+k}^{1}, G_{0} \pi_{J_{t+k}^{2}}\right]\right) \pi_{J_{t+k}^{1}, J} \neq 1 \text { for some } J \in \mathbf{n}^{s}, \\
\text { then } G_{J_{t+k-1}^{1-}} \cap\left(\operatorname{Soc}\left(G_{J_{t+k-1}^{2}}\right) \cap\left[N_{t+k-1}^{1}, G_{0} \pi_{J_{t+k-1}^{1}}^{1}\right]\right) \pi_{J_{t+k}^{1}, J} \neq 1 .
\end{array}\right.
$$

Suppose, $\left(\alpha_{t+1}\right)$ holds. We know that $J_{[2, t+1]}^{1} \in \mathscr{B}_{t}^{* *}$; in fact, $G_{0} \pi_{J_{t+1}^{1}}=G_{j_{1}^{1}} \pi_{J_{t+1}^{1}}$, since $G_{j_{1}^{1}} \pi_{J_{t+1}^{1}} \cong G_{0} \pi_{j_{[2, t+1]}^{1}} \cong G_{0} \pi_{J_{t+1}^{1}}$ (Lemma 5.7). Hence

$$
G_{J_{t+1}^{1}} \cap\left(\operatorname{Soc}\left(G_{J_{t+1}^{1}}\right) \cap\left[N_{t+1}^{1}, G_{0} \pi_{J_{t+1}^{1}}\right]\right) \pi_{J_{t+1}^{1}, J} \neq 1
$$

if and only if $G_{J_{[2, t+1]}^{1}} \cap\left(\operatorname{Soc}\left(G_{J_{[2, t+1]}^{1}}\right) \cap\left[N_{[2, t+1]}^{1}, G_{0} \pi_{J_{[2, t+1]}^{1}}\right]\right) \pi_{J_{[2, t+1]}^{1}} \neq 1$, where

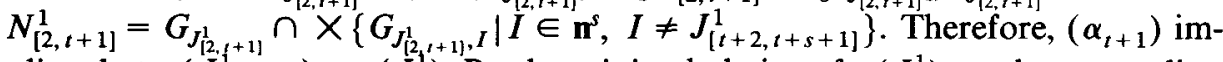
plies that $c\left(J_{[2, t+1]}^{1+1}\right) \leqslant c\left(J_{t}^{1}\right)$. By the minimal choice of $c\left(J_{t}^{1}\right)$, we have equality. This means that all $J_{s}^{j} \in \mathscr{C}\left(J_{t}^{1}\right)$ begin with $j_{t+1}^{1}$.

Proceeding in the same manner, we see that in presence of $\left(\alpha_{t+1}\right), \ldots,\left(\alpha_{t+i}\right)$ ( $i \in\{1, \ldots, s\}$ ), all $J_{s}^{j} \in \mathscr{C}\left(J_{t}^{1}\right)$ begin with $\left(j_{t+1}^{1}, \ldots, j_{t+i}^{1}\right)$. In particular, if $\left(\alpha_{t+1}\right), \ldots,\left(\alpha_{t+s}\right)$ are fulfilled, then $J \in \mathscr{C}\left(J_{t}^{1}\right)$ if and only if $J=J_{[t+1, t+s]}^{1}$. But $\left[N_{t}^{1}, G_{0} \pi_{J_{t}^{1}}\right] \leqslant N_{t}^{1}$, whence $\left[N_{t}^{1}, G_{0} \pi_{J_{t}^{1}}\right] \pi_{J_{t}^{1}, J_{t+1, t+s]}^{1}}=1$ by definition of $N_{t}^{1}$. However, this contradicts the definition of $\mathscr{C}\left(J_{t}^{1}\right)$. Consequently, there exists some $w \in\{1, \ldots, s\}$ for which $\left(\alpha_{t+w}\right)$ fails to hold. If we set $r=t+w-1$, then assertion (3) follows.

(4) Let $r$ and $J_{s}^{k} \in \mathscr{B}_{s}$ be as in (3). Then

$$
G_{J_{r}^{1}} \cap\left(\operatorname{Soc}\left(G_{j_{r}^{1}}\right) \cap\left[N_{r+1}^{1}, G_{0} \pi_{J_{r+1}^{1}}, N_{r}^{1}\right]\right) \pi_{J_{r+1}^{1}, J_{s}^{k}} \neq 1 .
$$

Since $\left[N_{r+1}^{1}, G_{0} \pi_{J_{r+1}^{1}}\right] \leqslant\left[G_{J_{r+1}^{1}}, G_{J_{r}^{1}} \pi_{J_{r+1}^{1}}\right] \leqslant G_{J_{r}^{1}}$ by 3.3(b), it follows from (3) that there exists a minimal normal subgroup $M$ of $G_{J_{r}^{1}}$ such that $M \leqslant\left[N_{r+1}^{1}, G_{0} \pi_{J_{r+1}^{1}}\right]$ and $M \pi_{J_{r+1}^{1}, J_{s}^{k}} \neq 1$. By 5.13, $M \pi_{J_{r+1}^{1}, J_{s}^{k}} \leqslant G_{J_{r}^{1}}$. Now every prime divisor of $\left\|\left[N_{r+1}^{1}, G_{0} \pi_{J_{r+1}^{1}}\right]\right\|$ is also a divisor of $\mid G_{0} \pi_{J_{r+1}^{1}} /$ Core $_{G_{J_{r+1}^{1}}}\left(G_{0} \pi_{J_{r+1}^{1}}\right) \mid$, and hence of $\left|G / G^{\prime}\right|$. Since $\left(\left|G / G^{\prime}\right|,|Z(G)|\right)=1$, it follows that $M \pi_{J_{r+1}^{1}, J_{s}^{k}} \nless Z\left(G_{J_{r}^{1}}\right)$. Using the 
fact that $G_{0} \pi_{J_{r}^{1}}$ is subnormal in $G_{J_{r}^{1}}$ of defect at most $r$, we conclude that $\left[M \pi_{J_{r+1}^{1}, J_{s}^{k}}, G_{0} \pi_{J_{r}^{1}}, \ldots, G_{0} \pi_{J_{r}^{\prime}}\right] \leqslant\left[G_{J_{r}^{1}}, G_{0} \pi_{J_{r}^{1}}, \ldots, G_{0^{\prime}} \pi_{J_{r}^{1}}\right] \leqslant G_{0} \pi_{J_{r}^{1}}$ (here $G_{0} \pi_{J_{r}^{1}}$ occurs $r$ times). On the other hand, this iterated commutator is contained in $G_{J_{r+1}^{1}, J_{s}^{k}}$. According to 3.3(e), $G_{J_{r}^{1}} \cap G_{J_{r_{+}}^{1}, J_{s}^{k}} \neq 1$ if and only if $\left(j_{r+1}^{1}, J_{s}^{k}\right) \in \mathscr{B}_{s+1}$. Hence, if $G_{0} \pi_{J_{r}^{1}} \cap G_{J_{r+1}^{1}, J_{s}^{k}} \neq 1$ then $\left(J_{r+1}^{1}, J_{s}^{k}\right)=J_{r+s+1}^{1}\left(\right.$ note that $\left.G_{0} \pi_{J_{r}^{1}} \cap N_{r}^{1}=1\right)$. But this would lead to the contradiction $M \pi_{J_{r+1}^{1}, J_{s}^{k}}=M \pi_{J_{r+s+1}^{1}} \leqslant N_{r+1}^{1} \pi_{J_{r+s+1}^{1}}=1$. Thus $\left[M \pi_{J_{r+1}^{1}, J_{s}^{k}}, G_{0} \pi_{J_{r}^{1}}, \ldots, G_{0} \pi_{J_{r}^{1}}\right]=1$. But then $\left[M, N_{r}^{1}\right] \pi_{J_{r+1}^{1}, J_{s}^{k}}=\left[M \pi_{J_{r+1}^{1}, J_{s}^{k}}, N_{r}^{1}\right] \neq 1$, for otherwise the minimal normal subgroup $M_{J_{J_{r+1}}^{1}, J_{s}^{k}}$ of $G_{J_{r}^{1}}=N_{r}^{1} \cdot G_{0} \pi_{J_{r}^{1}}$ would be central. This proves (4).

(5) Let $r$ and $J_{s}^{k} \in \mathscr{B}_{s}$ be as in (3). Then

$$
G_{J_{r}^{1}} \cap\left(\operatorname{Soc}\left(G_{J_{r}^{1}}\right) \cap\left[N_{r}^{1}, G_{0} \pi_{J_{r}^{1}}\right]^{G_{J_{r+1}^{1}}}\right) \pi_{J_{r+1}^{1}, J_{s}^{k}} \neq 1 .
$$

It follows from the Three Subgroups Lemma [4, III.1.4] that

$$
\left[N_{r+1}^{1}, G_{0} \pi_{J_{r+1}^{1}}, N_{r}^{1}\right]=\left[N_{r+1}^{1}, G_{0} \pi_{J_{r}^{1}}, N_{r}^{1}\right] \leqslant\left[N_{r}^{1}, G_{0} \pi_{J_{r}^{1}}\right]^{G_{J_{r+1}^{1}}}
$$

because $N_{r}^{1}$ is normalized by $N_{r+1}^{1}$. The assertion follows from (4).

(6) With $G_{J_{r}^{1}}, N_{r}^{1}, G_{0} \pi_{J_{r}^{1}}$ in place of $G, N, S$, statements (a)-(e) of the theorem are fulfilled.

Apart from the non-nilpotency of $N_{r}^{1}$, assertions (a), (b), (c) are clear from the considerations in Section 5. That $N_{r}^{1}$ is not nilpotent is a simple consequence of $\left(\left|G / G^{\prime}\right|,|Z(G)|\right)=1$ : minimal normal subgroups of $G_{J_{r}^{1}}$ contained in $\left[N_{r}^{1}, G_{0^{\prime}} \pi_{J_{r}^{\prime}}\right]$ are central in $G_{J_{r}^{1}}$ in case $N_{r}^{1}$ (and hence $G_{J_{r}^{1}} / \operatorname{Core}_{G_{J_{1}^{1}}}\left(G_{0} \pi_{J_{r}^{1}}\right)$ ) is nilpotent. By (5), $\left[N_{r}^{1}, G_{0} \pi_{J_{r}^{1}}\right] \pi_{J_{r+1}^{1}, J_{s}^{k}} \neq 1$. If $\left[N_{r}^{1}, G_{0} \pi_{J_{r}^{1}}\right]$ is contained in $\operatorname{Soc}\left(G_{J_{r}^{\prime}}\right)$, then 5.13 yields $G_{J_{r}^{1}} \cap\left(\operatorname{Soc}\left(G_{J_{r}^{1}}\right) \cap\left[N_{r}^{1}, G_{0} \pi_{J_{r}^{1}}\right]\right) \pi_{J_{r+1}^{1}, J_{s}^{k}} \neq 1$, contradicting (3). The same contradiction arises directly from (5) if $\left[N_{r}^{1}, G_{0} \pi_{J_{r}}\right]$ is assumed to be invariant under $G_{J_{r+1}^{1}}$ (or even characteristic in $G_{J_{r}^{1}}$ ). This shows that (d) and (e) hold, thus completing the proof.

Let $G$ be a group with finite composition series. Theorem 6.2 shows that leaving condition (2) in Hawkes' conjecture as it stands while strengthening condition (1) in such a way that, apart from direct decompositions, factorizations as in Theorem 6.2 are also not allowed, forces $G$ to be normally detectable. In Theorem 6.5 we prove that it is also possible to strengthen condition (2) in such a way that directly indecomposable groups are then normally detectable.

To this end we introduce the following definition.

6.3. Definition. Let $G$ be a group. A factor group $\bar{G}$ of $G$ is called essential if either $\bar{G}=G$, or if the following conditions are satisfied:

(1) $\bar{G}$ is not nilpotent;

(2) $\bar{G}$ is directly decomposable (in a non-trivial way);

(3) $\bar{G}$ is isomorphic to a subnormal subgroup of $G$. 
Theorem 6.5 will be a consequence of the following lemma, where again the notation of 5.2 is used.

6.4. Lemma. Let $G$ be a group with finite composition series. Assume that $G_{0} \unlhd G_{1} \times \cdots \times G_{n}$, where $G_{j} \cong G$ for $j=0,1, \ldots, n$. Assume further that there exists some $J=J_{l}=\left(j_{1}, \ldots, j_{l}\right) \in \mathbf{n}^{\prime}(l \geqslant 2)$ such that the following conditions are satisfied:

(1) $G_{J}=N \cdot G_{0} \pi_{J}$, where $N \unlhd G_{J}$ and $N \cap G_{0} \pi_{J}=1$;

(2) $G_{0} \pi_{J} \unlhd G_{J_{i}} \pi_{J}$ for some $i \in\{1, \ldots, l-1\}$, where $J_{i}=\left(j_{1}, \ldots, j_{i}\right)$. Then $G_{0} \pi_{J} \unlhd G_{J_{i+1}} \pi_{J}$, or $\left(\left|Z\left(G_{J_{i}} \pi_{J}\right)\right|,\left|G_{J_{i}} \pi_{J} /\left(G_{J_{I}} \pi_{J}\right)^{\prime}\right|\right) \neq 1$. Moreover, if $i=l-1$, then $G_{0} \pi_{J} \unlhd G_{J}$, or $\left(|Z(G)|,\left|G / G^{\prime}\right|\right) \neq 1$.

PRoof. Note that $Z\left(G_{J_{i}} \pi_{J}\right)$ and $G_{J_{i}} \pi_{J} /\left(G_{J_{i}} \pi_{J}\right)^{\prime}$ are finite. Clearly, $G_{J_{i}} \pi_{J} \unlhd G_{J_{i+1}} \pi_{J}$. Using the modular law we conclude from (1) and (2) that $G_{J_{i}} \pi_{J}=\left(N \cap G_{J_{i}} \pi_{J}\right) \times$ $G_{0} \pi_{J}$. Now, $\left[N \cap G_{J_{i+1}} \pi_{J}, G_{0} \pi_{J}\right] \leqslant N \cap G_{J_{i}} \pi_{J}$. Since, for any $n \in N \cap G_{J_{i+1}} \pi_{J}$, $G_{0} \pi_{J}$ and $\left(G_{0} \pi_{J}\right)^{n}$ centralize $N \cap G_{J_{I}} \pi_{J}$, it follows that $\left[N \cap G_{J_{i+1}} \pi_{J}, G_{0} \pi_{J}\right] \leqslant$ $Z\left(G_{J_{i}} \pi_{J}\right)$. Thus, if $G_{0} \pi_{J}$ is not normal in $G_{J_{t+1}} \pi_{J}=\left(N \cap G_{J_{i+1}} \pi_{J}\right) \cdot G_{0} \pi_{J}$, there exists some $n \in N \cap G_{J_{i+1}} \pi_{J}$ such that $1 \neq\left[n, G_{0} \pi_{J}\right] \subseteq Z\left(G_{J_{i}} \pi_{J}\right)$. But then $\varphi: G_{J_{i}} \pi_{J} \rightarrow Z\left(G_{J_{i}} \pi_{J}\right)$, defined by $(m x) \varphi=[n, x]$ for $m \in G_{J_{i}} \pi_{J} \cap N$ and for $x \in G_{0} \pi_{J}$, is a non-trivial homomorphism. This proves the first part of the lemma.

Now let $i=l-1$. We have shown that $1 \neq\left[n, G_{0} \pi_{J}\right] \subseteq Z\left(G_{J_{t-1}} \pi_{J}\right)$ for some $n \in N$, unless $G_{0} \pi_{J} \unlhd G_{J}$. If $\left[n, G_{0} \pi_{J}\right] \subseteq Z\left(G_{J}\right)$, then $\pi_{J} \varphi$ is a non-trivial homomorphism from $G_{J_{t-1}}$ into $Z\left(G_{J}\right)$, where $\varphi$ is defined as above. Hence $\left(|Z(G)|,\left|G / G^{\prime}\right|\right) \neq 1$. So we may assume that there exists some $y \in G_{J}$ such that $\left[G_{0} \pi_{J}, n, y\right] \neq 1$. Since $Z\left(G_{J_{t-1}} \pi_{J}\right) \unlhd G_{J}$, it follows that $\left[G_{0} \pi_{J}, n, y\right] \subseteq$ $Z\left(G_{J_{t-1}} \pi_{J}\right) \cap G_{J_{I-1}} \leqslant Z\left(G_{J_{t-1}}\right)$. Then $\rho: G_{J_{t-1}} \pi_{J} \rightarrow Z\left(G_{J_{l-1}}\right)$, defined by $(m x) \rho=$ $[x, n, y]$ for $x \in G_{0} \pi_{J}$ and for $m \in G_{J_{l-1}} \pi_{J} \cap N$, is a non-trivial homomorphism; this yields $\left(|Z(G)|,\left|G / G^{\prime}\right|\right) \neq 1$ again.

6.5. THEOREM. Let $G$ be a directly indecomposable group with finite composition series. Assume that $\left(|Z(\bar{G})|,\left|\bar{G} / \bar{G}^{\prime}\right|\right)=1$ for all essential factor groups $\bar{G}$ of $G$. Then $G$ is normally detectable.

Proof. By way of contradiction, we assume that $G$ is not normally detectable. Since $\left(|Z(G)|,\left|G / G^{\prime}\right|\right)=1, G$ is not nilpotent. We choose $J_{l}=\left(j_{1}, \ldots, j_{l}\right)$ as in Theorem 5.10 and note that (a) $-(\mathrm{g})$ of 5.10 are satisfied. For $1 \leqslant i \leqslant l$, let $J_{i}=\left(j_{1}, \ldots, j_{i}\right)$. We now prove by induction on $i(1 \leqslant i \leqslant l)$ that

$$
G_{J_{i}} \pi_{J_{l}}=\left(G_{J_{l}} \pi_{J_{l}} \cap N_{J_{l}}\right) \times G_{0} \pi_{J_{l}}
$$

where $N_{J_{l}}=G_{J_{l}} \cap \times\left\{G_{J_{l}, J} \mid J \in \mathbf{n}^{l}, J \neq J_{l}\right\}$. The case $i=l$ then yields a contradiction to the assumption that $G_{J_{t}}$ is directly indecomposable (note 5.10(c), (d)). Clearly, by the modular law and by parts (a) and (b) of $5.10, G_{J_{i}} \pi_{J_{I}}$ is a 
semidirect product of the normal subgroup $G_{J_{I}} \pi_{J_{I}} \cap N_{J_{I}}$ and the subnormal subgroup $G_{0} \pi_{J_{i}}$. Since $G_{0} \pi_{J_{l}} \unlhd G_{j_{1}} \pi_{J_{l}},(*)$ holds for $i=1$. Let $1<i \leqslant l$ and suppose that (*) holds for $i-1$. Employing 6.4, we see that either $(*)$ holds for $i$, or that $\left(\left|G_{J_{i-1}} \pi_{J} /\left(G_{J_{i-1}} \pi_{J_{l}}\right)^{\prime}\right|,\left|Z\left(G_{J_{i-1}} \pi_{J}\right)\right|\right) \neq 1$. But the latter alternative is impossible since, by induction hypothesis, $G_{J_{i-1}} \pi_{J_{l}}$ is an essential factor group of $G_{J_{i-1}} \cong G$. This completes the induction argument.

In the sequel we are going to enlarge our knowledge of the type of embedding of a possible counterexample to Hawkes' conjecture. For the proof of Theorem 6.8 , which contains the relevant facts, two lemmas are needed.

General hypothesis for 6.6 and 6.7: $G$ denotes a non-trivial directly indecomposable group with finite composition series, where we assume that $\left(\left|G / G^{\prime}\right|,|Z(G)|\right)=1$. In particular, $G$ is not nilpotent. Let $G_{0} \unlhd G_{1} \times \cdots \times G_{n}$ be a non-trivial embedding, where $n \geqslant 2$, where $G_{j} \cong G$ for $j=0,1, \ldots, n$, and where $G_{0} \pi_{i} \neq 1$ for $i=1, \ldots, n$. Moreover, we use the notation from 5.2.

6.6. LEMMA. Let $r$ be a positive integer and let $J_{r}=\left(j_{1}, \ldots, j_{r}\right) \in \mathbf{n}^{r}$. Suppose that $\left\{I_{l(i)}\right\}_{i=1}^{t}$ is a (lexicographically ordered) set of $l(i)$-tuples, $l(i) \in \mathbb{N}, i=$ $1, \ldots, t, t \in \mathbb{N}$, such that $G_{0} \leqslant X_{i=1}^{t} G_{I_{(i)}}$. For those $i \in\{1, \ldots, t\}$ for which $G_{0} \pi_{\left(j_{2}, \ldots, j_{r}, I_{h i}\right)}$ is not nilpotent, assume that there exists $m(i) \in \mathbb{N}$ and $K_{m(i)} \in \mathbf{n}^{m(i)}$

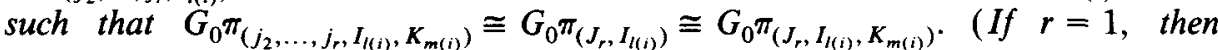
$G_{0} \pi_{\left(j_{2}, \ldots, j_{r}, I\right)}$ stands for $\left.G_{0} \pi_{I}.\right)$ Then $Z_{\infty}\left(G_{0} \pi_{J_{r}}\right) \leqslant Z_{\infty}\left(G_{j_{1}} \pi_{J_{r}}\right)$.

Proof. If $G_{0} \pi_{\left(j_{2}, \ldots, j_{r}, I_{l(i)}\right)}$ is not nilpotent, then, putting $N_{r, l(i)}=G_{J_{r}, I_{l(i)}} \cap$

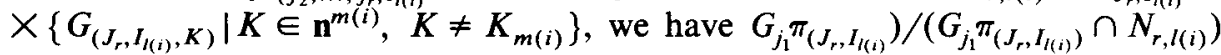
$\cong G_{j_{1}} \pi_{\left(J_{r}, I_{(i)}, K_{m(i)}\right)} \cong G_{0} \pi_{\left(J_{r}, I_{(i)}\right)}$ by the assumed properties of $K_{m(i)}$. Similarly, $G_{0} \pi_{\left(J_{r}, I_{l i}\right)} /\left(G_{0} \pi_{\left(J_{r}, I_{l(i)}\right)} \cap N_{r, l(i)}\right) \cong G_{0} \pi_{\left(J_{r}, I_{l(i)}, K_{m(i)}\right)} \cong G_{0} \pi_{\left(J_{r}, I_{(i)}\right)}$. It follows that $G_{0} \pi_{\left(J_{r}, I_{l i}\right)} \cap N_{r, l(i)}=1$ and that $G_{j_{1}} \pi_{\left(J_{r}, I_{l(i)}\right)}=\left(G_{j_{1}} \pi_{\left(J_{r}, I_{l i}\right)} \cap N_{r, l(i)}\right) \times G_{0} \pi_{\left(J_{r}, I_{(i)}\right)}$. This implies that $Z_{\infty}\left(G_{0} \pi_{\left(J_{r}, I_{(i)}\right)}\right) \leqslant Z_{\infty}\left(G_{j_{1}} \pi_{\left(J_{r}, I_{(i)}\right)}\right)$. The same statement holds trivially if $G_{j_{1}} \pi_{\left(J_{r}, I_{I(i)}\right)}$ is nilpotent. We conclude that $Z_{\infty}\left(G_{0} \pi_{J_{r}}\right) \leqslant$ $\left(\times_{i=1}^{t} Z_{\infty}\left(G_{0} \pi_{\left(J_{r}, I_{(i)}\right)}\right)\right) \cap G_{j_{1}} \pi_{J_{r}} \leqslant\left(\times_{i=1}^{t} Z_{\infty}\left(G_{j_{1}} \pi_{\left(J_{r}, I_{(i)}\right)}\right)\right) \cap G_{j_{1}} \pi_{J_{r}} \leqslant Z_{\infty}\left(G_{j_{1}} \pi_{J_{r}}\right)$.

6.7. Lemma. Let $J^{i}=\left(j_{1}^{i}, j_{2}^{i}, \ldots\right) \in \mathscr{B}_{\infty}$ for some $i \in\{1, \ldots, b\}$. Suppose that $j=j_{1}^{i} \neq j_{1}^{h}$ for all $h \in\{1, \ldots, b\}, h \neq i$. Then the following statements hold.

(a) $j_{l}^{i} \neq j$ for all $l>1$.

(b) $s(i)=\cdot 1$, i.e. $G_{0} \pi_{J^{i}} \cong G_{0} \pi_{J^{i}}$ for all $l \geqslant 1$.

Proof. (a) Suppose the assertion is false. If $r \geqslant 1$ is an integer such that $j_{r+1}^{i}=j$, then it follows from the hypothesis and from 5.4 that $j_{k}^{i}=j_{k+m r}^{i}$ for all positive integers $k$ and $m$. Hence we may choose such an integer $r$ with $r \geqslant s$ 
(where $s$ is the stationary level of the iterated embedding of $G_{0}$; cf. 5.2(d)). Let $1 \leqslant k \leqslant r-1$ be arbitrary. If, for some $I \in \mathbf{n}^{k}, G_{0} \pi_{\left(j_{k+2}^{i}, \ldots, j_{r+1}^{i}, I\right)}$ is not nilpotent, then, by $5.4, G_{0} \pi_{\left(j_{r+1}^{i}, I\right)}$ is not nilpotent (note that $k+2 \leqslant r+1$ ). Since $j_{r+1}^{i}=j$, we deduce from the hypothesis that $I=\left(j_{2}^{i}, \ldots, j_{k+1}^{i}\right)=\left(j_{r+2}^{i}, \ldots, j_{r+k+1}^{i}\right)$. Let $\sigma$ denote the $r$-cycle $(1, \ldots, r)$ in the symmetric group $S_{r}$. Then 5.5 (c) yields $G_{0} \pi_{\left(j_{k+2}^{i}, \ldots, j_{r+1}^{i}, I, j_{r+k+2}^{i}\right)}=G_{0} \pi_{\left(J_{r}^{i} \boldsymbol{o}_{1}^{k+1}, j_{k+2}^{i}\right)} \cong G_{0} \pi_{J_{r}^{\prime}}, \quad G_{0} \pi_{\left(j_{k+1}^{i}, \ldots, j_{r+1}^{i}, I\right)}=G_{0} \pi_{\left(J_{r}^{i} \mathbf{o}^{k}, j_{k+1}^{i}\right)}$ $\cong G_{0} \pi_{J_{r}^{i}}$, and $G_{0} \pi_{\left(j_{k+1}^{i}, \ldots, j_{r+1}^{i}, I, j_{r+k+2}^{i}\right)}=G_{0} \pi_{\left(J_{r}^{i} \sigma^{k}, j_{k+1}^{i}, j_{k+2}^{i}\right)} \cong G_{0} \pi_{J_{r}^{i}}$. Hence Lemma 6.6 is applicable. We conclude that

$$
Z_{\infty}\left(G_{J_{k}^{i}} \pi_{J_{r+1}^{i}}\right) \leqslant Z_{\infty}\left(G_{J_{r}} \pi_{J_{r+1}^{i}}\right) \text { for all } 1 \leqslant k \leqslant r .
$$

Moreover, a similar argument as above shows that if $\left(j_{t}^{i}, \ldots, j_{t+u}^{i}, J\right) \in \mathscr{B}_{u+r+1}$, where $t \geqslant 1, u \geqslant r-1$, and $J \in \mathbf{n}^{r}$, then $J=\left(j_{t+u+1}^{i}, \ldots, j_{t+u+r}^{i}\right)$ and $G_{0} \pi_{\left(j_{t}^{i}, \ldots, j_{t+u}^{i}\right)} \cong G_{0} \pi_{\left(j_{t}^{i}, \ldots, j_{t+r-1}^{i}\right)} \cong G_{0} \pi_{J}(5.5(\mathrm{c}))$. In particular, $J_{r+1}^{i} \in \mathscr{B}_{r+1}^{*}$. It follows from 5.5(a) that $G_{J_{r+1}^{i}}$ is a semidirect product of $N_{r+1}^{l}$ and $G_{0} \pi_{J_{r+1}^{i}}$.

We now aim to apply Lemma 6.4. Clearly, $G_{0} \pi_{J_{r+1}^{i}} \unlhd G_{j_{1}^{\prime}} \pi_{J_{r+1}^{i}}$. Let $1 \leqslant k \leqslant r$. If $\left(\left|G_{J_{k}} \pi_{J_{r+1}^{i}} /\left(G_{J_{k}^{i}} \pi_{J_{r+1}^{i}}\right)^{\prime}\right|,\left|Z\left(G_{J_{k}} \pi_{J_{r+1}^{i}}\right)\right|\right) \neq 1$, then, by means of $(*)$ and the fact that the prime divisors of $\left|Z_{\infty}\left(G_{J_{r}} \pi_{J_{r+1}^{i}}\right)\right|$ and those of $\left|Z\left(G_{J_{r}} \pi_{J_{r+1}^{i}}\right)\right|$ coincide, there exists a non-trivial homomorphism $\varphi$ from $G_{J_{k}^{i}}$ into $Z\left(G_{J_{r}^{i}} \pi_{J_{r+1}^{i}}\right)$. Now, $\left[G_{J_{k}} \varphi, G_{J_{r+1}^{i}}\right] \leqslant Z\left(G_{J_{r}^{i}} \pi_{J_{r+1}^{i}}\right) \cap G_{J_{r}^{i}} \leqslant Z\left(G_{J_{r}^{i}}\right)$. Hence, if $\left[G_{J_{k}^{i}} \varphi, g\right] \neq 1$ for some $g \in$ $G_{J_{r+1}^{i}}$, then $\rho: G_{J_{k}^{i}} \varphi \rightarrow Z\left(G_{J_{r}^{\prime}}\right)$, defined by $y \rho=[y, g]$ for all $y \in G_{J_{k}^{i}} \varphi$, is a non-trivial homomorphism, contradicting $\left(\left|G / G^{\prime}\right|,|Z(G)|\right)=1$. Therefore, $G_{J_{k}^{\prime}} \varphi$ $\leqslant Z\left(G_{J_{r+1}^{i}}\right)$. But this leads to the same contradiction. Consequently, $\left(\left|G_{J_{k}} \pi_{J_{r+1}^{i}} /\left(G_{J_{k}^{i}} \pi_{J_{r+1}^{i}}\right)^{\prime}\right|,\left|Z\left(G_{J_{k}} \pi_{J_{r+1}^{i}}\right)\right|\right)=1$ for all $1 \leqslant k \leqslant r$. So we apply Lemma 6.4 to deduce that $G_{0} \pi_{J_{r+1}^{i}} \unlhd G_{J_{r+1}^{i}}$. Thus $G_{J_{r+1}^{i}}=N_{r+1}^{i} \times G_{0} \pi_{J_{r+1}^{i}}$. Since the embedding of $G_{0}$ in $G_{1} \times \cdots \times G_{n}$ is non-trivial, this contradicts the hypothesis that $G$ is directly indecomposable.

(b) Let $l \geqslant 1$ be arbitrary and set $N_{1, l}=G_{j_{1}^{i}} \cap \times\left\{G_{\left(j_{1}^{i}, J\right)} \mid J \in \mathbf{n}^{l-1}, J \neq J_{[2, l]}^{i}\right\}$. By hypothesis, $G_{0} \pi_{j_{1}} /\left(G_{0} \pi_{j_{1}^{\prime}} \cap G_{J_{i}^{i}}\right) \cong G_{0} \tilde{\pi}$ is nilpotent, where $\tilde{\pi}=\sum\left\{\pi_{\left(j_{1}^{i}, J\right)} \mid\right.$ $\left.J \in \mathbf{n}^{l-1}, J \neq J_{[2, l]}^{i}\right\}$. It follows from 3.3(e) that $G_{0} \cap N_{1, l}=1$. This implies that $G_{0} \pi_{j_{1}} \cap N_{1, l}$ is central in $G_{j_{1}}$. If this group is non-trivial, then there exists a non-trivial homomorphism from the nilpotent group $G_{0} \pi_{j i} /\left(G_{0} \pi_{j_{1}^{i}} \cap G_{J_{i}}\right)$ into $\left(\left(G_{0} \pi_{j_{1}} \cap N_{1, l}\right) \times\left(G_{0} \pi_{j_{1}^{i}} \cap G_{J_{i}}\right)\right) /\left(G_{0} \pi_{j^{i}} \cap G_{J_{i}}\right) \cong G_{0} \pi_{j_{1}} \cap N_{1, l} \leqslant Z\left(G_{j_{1}^{\prime}}\right)$ (Lemma 3.2). This contradicts $\left(\left|G / G^{\prime}\right|,|Z(G)|\right)=1$. Consequently, $G_{0} \pi_{j^{i}} \cap N_{1, l}=1$, i.e. $G_{0} \pi_{j_{1}^{i}}=G_{0} \pi_{J_{i}^{i}}$.

6.8. TheOrem. Let $G$ be a group with finite composition series. Assume that $G$ is directly indecomposable and that $\left(\left|G / G^{\prime}\right|,|Z(G)|\right)=1$. If $G$ is not normally detectable, and if $G_{0} \unlhd G_{1} \times \cdots \times G_{n}$ (where $n \geqslant 2, G_{j} \cong G$ for $j=0,1, \ldots, n$, and $G_{0} \pi_{i} \neq 1$ for $\left.i=1, \ldots, n\right)$ is a non-trivial embedding, then the following hold.

(a) The stationary level s of the iterated embedding is at least 3.

(b) The branching number $b$ of the iterated embedding is at least $n+1$. In particular, $b \geqslant 3$. 
(c) If $b=3$, then, up to interchanging the indices 1 and 2, there is only one possible embedding. This is of the following type: $\mathscr{B}_{\infty}=\left\{J^{1}, J^{2}, J^{3}\right\}$, where $J^{1}=(1,1,1,1, \ldots), J^{2}=(1,2,1,1, \ldots)$, and $J^{3}=(2,1,1,1, \ldots)$. Moreover, in this case $s(1)>2, s(2)=2, s(3)=1$ and $\mathscr{B}_{\infty}^{* *}=\left\{J^{1}\right\} ;$ in fact, $\mathscr{B}_{k}^{*}=\left\{J_{k}^{1}\right\}$ for all $k \geqslant s$.

Proof. (a) Using 5.8, we choose $J_{s}^{i} \in \mathscr{B}_{s}^{*}$. Then, by 5.5(a), $G_{J_{s}^{i}}$ is a semidirect product of $N_{s}^{i}$ and $G_{0} \pi_{J_{s}}$. Since the embedding is non-trivial, both factors are distinct from 1. The hypothesis that $G$ is directly indecomposable yields $s>1$. Moreover, $s=2$ is impossible by the last statement of Lemma 6.4.

(b) Clearly, $b \geqslant n$. By 3.3(e), (f), $G_{0} \pi_{i} \neq 1$ if and only if $G_{0} \pi_{i} \nless$ Fit $\left(G_{i}\right)$. Hence, if $b=n$, we may assumes that $J_{1}^{i}=(i)$ for $i=1, \ldots, n$. It follows from 6.7 that $s(i)=1$ for all $i$, i.e. that $s=1$. This contradicts part (a). Thus $b \geqslant n+1 \geqslant 3$.

(c) If $b=3$, then $n=2$ by part (b). By using 5.4 and 6.7 , it is easy to see that, up to interchanging the indices 1 and $2, J^{1}, J^{2}, J^{3} \in \mathscr{B}_{\infty}$ have to be of the form given above. By 6.7, $s(3)=1$. Hence $G_{0} \pi_{2} \cong G_{0} \pi_{\left(2, J_{l}^{1}\right)}$ for all $l \geqslant 1$. We conclude that $G_{1} \pi_{12} \cong G_{1} \pi_{\left(1,2, J_{l}^{l}\right)}$. This implies that $G_{0} \pi_{12} \cong G_{0} \pi_{\left(1,2, J_{l}^{1}\right)}$ for all $l \geqslant 1$, i.e. that $s(2) \leqslant 2$. If $s(2)=1$, then $G_{0} \pi_{1} \cong G_{0} \pi_{12} \unlhd G_{1} \pi_{12} \cong G_{0} \pi_{2}$, and, as $s(3)=1, G_{0} \pi_{2} \cong$ $G_{0} \pi_{21} \unlhd G_{2} \pi_{21} \cong G_{0} \pi_{1}$, i.e. $G_{0} \pi_{1} \cong G_{0} \pi_{2} \cong G_{0} \pi_{21}$. But this leads to a non-trivial decomposition $G_{2}=\left(G_{2} \cap G_{22}\right) \times G_{0} \pi_{2}$, which is a contradiction. Hence $s(2)=2$. Then $s(1)>2$ by part (a).

By 5.4, $G_{0} \pi_{J_{s}^{2}}$ and $G_{0} \pi_{J_{s}^{3}}$ are isomorphic to subnormal subgroups of $G_{0} \pi_{J_{s}^{1}}$. Therefore, $J_{k}^{1} \in \mathscr{B}_{k}^{*}$ for all $k \geqslant s$, according to 5.8. If $J_{k}^{i} \in \mathscr{B}_{k}^{*}$ for some $i \in\{2,3\}$, and if $k \geqslant s$, then, by definition of $\mathscr{B}_{k}^{*}, G_{0} \pi_{J_{k}} \cong G_{0} \pi_{J_{s}}$. Since $s(2)=2$ and $s(3)=1$, it follows that $G_{0} \pi_{12} \cong G_{0} \pi_{J_{s}^{1}}$ (in case $i=2$ ), or that $G_{0} \pi_{2} \cong G_{0} \pi_{J_{s}^{1}}$ (in case $i=3$ ). In the first case, let $N=G_{12} \cap \times\left\{G_{(1,2, J)} \mid J \in \mathbf{n}^{s}, J \neq J_{s}^{1}\right\}$. Then $G_{12} / N \cong G_{0} \pi_{J_{s}^{1}} \cong G_{0} \pi_{12}$, and $G_{0} \pi_{12} /\left(G_{0} \pi_{12} \cap N\right) \cong G_{0} \pi_{\left(1,2, J_{s}^{1}\right)} \cong G_{0} \pi_{12}$. It follows that $G_{12}$ is a semidirect product of $N$ and $G_{0} \pi_{12}$. The last statement of Lemma 6.4 now leads to a contradiction. The case $G_{0} \pi_{2} \cong G_{0} \pi_{J_{s}^{1}}$ is even easier and leads in a similar way to a non-trivial direct decomposition of $G_{2}$.

We have been unable to decide whether the situation in 6.8(c) can actually occur.

6.9. TheOREM. Let $G$ be a group with finite composition series. Assumes that $G$ is directly indecomposable and that $\left(\left|G / G^{\prime}\right|,|Z(G)|\right)=1$. Then $G$ is normally detectable, provided that one of the following conditions is satisfied.

(1) If $\alpha \in \operatorname{Aut}(G)$ is such that $\langle\alpha\rangle \cong H / M$ for some $H \leqslant G, M \unlhd H$, then $\alpha$ preserves the conjugacy classes in $G$.

(2) $G$ possesses a unique maximal normal subgroup. 
(3) $\operatorname{Soc}(G)$ is a direct product of at most two minimal normal subgroups of $G$ or of three minimal normal subgroups of $G$ which are not all elementary abelian of the same order.

(4) $\operatorname{Fit}(G) \leqslant \operatorname{Soc}(G)$.

(5) $G$ is finite and, for every prime divisor $p$ of $\left|G / G^{\prime}\right|$, the Sylow p-subgroups of $G$ are abelian.

Proof. (1) and (2) follow immediately from 6.2(e) and (a), respectively, and 6.2(c) and (d) imply (4). We note in passing that for case (1) a direct argument along the lines of the proof of Theorem 4.2 is also possible. This is because condition (1) implies that $G_{0}$ is normal in $\times_{J \in \mathrm{n}^{\prime}} G_{J}$ for every $l \in \mathbb{N}$.

From Theorem 6.8(b) we see that the first condition in (3) forces $G$ to be normally detectable. Moreover, if $G$ is not normally detectable, and if $\operatorname{Soc}(G)$ is a direct product of three minimal normal subgroups of $G$, then we are in the situation of Theorem 6.8(c). It follows that $G_{0} \cap G_{2}, G_{0} \cap G_{12}$, and $G_{0} \cap G_{11}$ each contain exactly one minimal normal subgroup of $G_{0}$. It is a straightforward matter to show that the minimal normal subgroup of $G_{0}$ contained in $G_{0} \cap G_{2}$ $\left(G_{0} \cap G_{12}\right)$ coincides with the minimal normal subgroup of $G_{2}\left(G_{1}\right)$ contained in $G_{2} \cap G_{211}\left(G_{1} \cap G_{12}\right)$. This implies that all minimal normal subgroups of $G$ are isomorphic. Since at least one of them is abelian (Theorem 6.2(d)), we have the situation which is excluded in (3).

Finally, because $G$ is not nilpotent or $G=1$, (5) follows immediately from 5.11

\section{Concluding remarks}

\section{A. Characteristic embeddings}

The method of iterated embeddings works smoothly for transitive relations such as subnormality. So it is no surprise that this idea provides a short proof for the following result on characteristic embeddings.

7.1. Proposition. Let $G$ be a group satisfying the minimal or maximal condition on normal subgroups. If $G_{0}$ is a characteristic subgroup of $G_{1} \times \cdots \times G_{n}$, where $n \geqslant 2$, and $G_{j} \cong G$ for $j=0,1, \ldots, n$, then $G=1$.

Proof. If $G_{0} \cap G_{i}=1$ for some $i$, then $G_{0} \cap G_{j}=1$ for all $j=1, \ldots, n$, since the symmetric group $S_{n}$ acts in a natural way on $G_{1} \times \cdots \times G_{n}$. By 3.3(c), $G_{0} \pi_{j}$ is centralized by every automorphism of $G_{j}$. It follows easily that $G$ is an elementary 
abelian 2-group. But then $\operatorname{Aut}\left(G_{1} \times \cdots \times G_{n}\right)$ acts transitively on the set of non-trivial elements of $G_{1} \times \cdots \times G_{n}$. Now any of the assumed finiteness conditions forces $G$ to be trivial.

So assume that $G_{0} \cap G_{j} \neq 1$ for all $j$. In the notation of 5.2(a), $G_{0}$ is embedded as a characteristic subgroup in $\times_{J \in \mathbb{N}^{\prime}} G_{J}, G_{J} \cong G$, for any positive integer $l$. By the argument given above, $G_{0} \cap G_{J} \neq 1$ for all $J \in \mathbf{n}^{\prime}$. Hence $G_{0}$ contains direct products of normal subgroups of unbounded length, which contradicts both the minimal and maximal condition on normal subgroups.

\section{B. Central products}

The following proposition shows that Theorem 4.2 can be extended to the more general case of central products instead of direct products, at least for groups admitting no non-trivial central extensions.

We recall that a group $G$ is a central product of the normal subgroups $N_{1}, \ldots, N_{k}$ if $G=N_{1} \cdots N_{k}$ and $\left[N_{i}, N_{j}\right]=1$ for all $i, j=1, \ldots, k, i \neq j$.

7.2. Proposition. Let $G$ be a group without non-trivial central extensions. If $G$ is subnormally (normally) detectable, then the following hold: whenever $G_{0}$ is subnormal (normal) in $G_{1} \cdots G_{n}$, where $G_{j} \cong G$ for $j=0,1, \ldots, n$, and where $G_{1} \cdots G_{n}$ is a central product of $G_{1}, \ldots, G_{n}$, then $G_{0}=G_{i}$ for some $i \in\{1, \ldots, n\}$.

Proof. Let $G_{0}$ be subnormal (normal) in $G_{1} \cdots G_{n}$. The map $\varphi: G_{1} \times \cdots \times G_{n} \rightarrow G_{1} \cdots G_{n}$ defined by $\left(g_{1}, \ldots, g_{n}\right) \varphi=g_{1} \cdots g_{n}$ is onto, and $\operatorname{ker} \varphi \leqslant Z\left(G_{1} \times \cdots \times G_{n}\right)$. Let $G_{0} \varphi^{-1}$ denote the full preimage of $G_{0}$ in $G_{1} \times \cdots \times G_{n}$. By assumption, $G_{0} \varphi^{-1}=K \times \operatorname{ker} \varphi$, where $K \cong K \varphi=G_{0}$. Clearly, $G_{0} \varphi^{-1}$ is subnormal (normal) in $G_{1} \times \cdots \times G_{n}$. If $G$ is subnormally detectable, then $K \unlhd G_{0} \varphi^{-1} \unlhd \unlhd G_{1} \times \cdots \times G_{n}$ implies $K=G_{i}$ for some $i$. Consequently, $G_{0}=G_{i}$. Now assume that $G$ is normally detectable and that $G_{0} \unlhd G_{1} \cdots G_{n}$. If $K$ is not normal in $G_{1} \times \cdots \times G_{n}$, there exists $g \in G_{1}$ $\times \cdots \times G_{n}$ such that $[K, g] \neq 1$. For $k \in K$ define $k \rho \in \operatorname{ker} \varphi$ by $[k, g]=k^{\prime} \cdot k \rho$, $k^{\prime} \in K$. Then $\rho: K \rightarrow \operatorname{ker} \varphi$ is a non-trivial homomorphism. From $\operatorname{ker} \varphi \leqslant$ $Z\left(G_{1} \times \cdots \times G_{n}\right)$ we deduce the existence of a non-trivial homomorphism from $G$ into $Z(G)$. But this contradicts the fact that $G$ is normally detectable (compare the remark at the beginning of Section 6). Hence $K \unlhd G_{1} \times \cdots \times G_{n}$, and the desired conclusion follows as in the case of subnormally detectable $G$.

\section{Lie algebras, associative rings}

The argument used to prove Theorem 4.2 is valid not only for groups. Instead of finding the most general version of 4.2 in the framework of universal algebra, we content ourselves with two important cases. 
We recall that for a (not necessarily associative) ring $R$ the Fitting radical Fit $(R)$ is defined to be the subring of $R$ generated by all nilpotent ideals of $R$. (Here and in the sequel, ideal means 2-sided ideal.)

Subideals are defined analogously to subnormal subgroups. (For associative rings, these are just the meta-ideals of finite index in the language of Baer [2].)

7.3. Theorem. Let $L$ be a Lie algebra over $R$, where $R$ is an associative and commutative ring with 1 . Assume that $L$ satisfies the minimal condition on subideals. Then the following statements are equivalent.

(i) Whenever $L_{0}$ is a subideal of $L_{1} \oplus \cdots \oplus L_{n}$, where $L_{j} \cong L$ for $j=0,1, \ldots, n$, then $L_{0}=L_{i}$ for some $i \in\{1, \ldots, n\}$.

(ii) $L$ is directly indecomposable (as a direct sum of ideals), and there exists no non-trivial homomorphism from $L$ into a nilpotent subideal of $L$.

Proof. One simply translates the proofs of Lemma 3.3(a) and Theorem 4.2 into the language of Lie algebras.

7.4. Corollary. Let $L$ be a Lie algebra over $K$, where $K$ is a field. Assume that $L$ satisfies the minimal condition on subideals. Then the following statements are equivalent.

(i) Whenever $L_{0}$ is a subideal of $L_{1} \oplus \cdots \oplus L_{n}$, where $L_{j} \cong L$ for $j=0,1, \ldots, n$, then $L_{0}=L_{i}$ for some $i \in\{1, \ldots, n\}$.

(ii) $L$ is directly indecomposable, and either $L$ is perfect (i.e. $L=[L, L]$ ) or there exists no non-trivial abelian subideal of $L$.

(In characteristic $0, L$ contains no non-trivial abelian subideals if and only if $\operatorname{Fit}(L)=0$.)

Proof. Because of the fact that, for char $K=0$, the Fitting radical is a nilpotent ideal which contains every nilpotent subideal of $L$ (see Amayo, Stewart [1, Theorem 6.2.1, Lemma 8.1.3]), the conclusion follows from 7.3.

7.5. THEOREM. Let $R$ be an associative ring which satisfies the minimal condition on subideals. Then the following statements are equivalent.

(i) Whenever $R_{0}$ is a subideal of $R_{1} \oplus \cdots \oplus R_{n}$, where $R_{j} \cong R$ for $j=0,1, \ldots, n$, then $R_{0}=R_{i}$ for some $i \in\{1, \ldots, n\}$.

(ii) $R$ is directly indecomposable, and there exists no non-trivial homomorphism from $R$ into Fit $(R)$. 
Proof. By a result of Baer [2, Corollary 5], every subring of Fit $(R)$ is a subideal of $R$ because $R$ satisfies the minimal condition on ideals. Moreover, it follows from [2, Proposition 8] that every nilpotent subideal of $R$ is contained in $\operatorname{Fit}(R)$. Now the argument of 4.2 is adaptable.

Although Hawkes' conjecture on normally detectable groups (and also the corresponding problem for Lie algebras) remains unsettled, the analogous situation in associative rings with unit can easily be handled.

7.6. Proposition. Let $R$ be an associative ring with unit. Assume that $R$ is indecomposable (as a direct sum of 2-sided ideals). If $R_{0}$ is a 2-sided ideal in $R_{1} \oplus \cdots \oplus R_{n}$, where $R_{j} \cong R$ for $j=0,1, \ldots, n$, then $R_{0}=R_{i}$ for some $i \in$ $\{1, \ldots, n\}$.

Proof. If $e$ is the unit of $R_{0}$, it is easily shown that $e$ is a central idempotent in $R_{1} \oplus \cdots \oplus R_{n}$. Consequently, $R_{0}$ is a direct summand of $R_{1} \oplus \cdots \oplus R_{n}$. But then $R_{0}=R_{i}$ for some $i$ (see Lambek [5, 1.4, Proposition 12]).

\section{References}

[1] R. K. Amayo and I. Stewart, Infinite-dimensional Lie algebras (Noordhoff, Leyden, 1974).

[2] R. Baer, 'Meta ideals' (Report of a conference on linear algebra (1956), Nat. Acad. Sci. USA, Washington D. C., 1957), pp. 33-52.

[3] K. Doerk and T. O. Hawkes, Finite soluble groups (in preparation).

[4] B. Huppert, Endliche Gruppen I (Springer, Berlin, 1967).

[5] J. Lambek, Lectures on rings and modules (Blaisdell, Waltham, Massachusetts, 1966).

[6] R. Remak, 'Über die Zerlegung der endlichen Gruppen in direkt unzerlegbare Faktoren', $J$. Reine Angew. Math. 139 (1911), 293-308.

[7] R. Remak, 'Über die Darstellung der endlichen Gruppen als Untergruppen direkter Produkte', J. Reine Angew. Math. 163 (1930), 1-44.

[8] D. J. S. Robinson, A course in the theory of groups (Springer, Berlin, 1982).

Mathematisches Institut

Albert-Ludwigs-Universität

Albertstraße $23 \mathrm{~b}$

D-7800 Freiburg

Federal Republic of Germany 